\title{
Construction of Parallel RIO Codes using Coset Coding with Hamming Codes
}

\author{
Akira Yamawaki, Hiroshi Kamabe and Shan Lu \\ Graduate School of Engineering \\ Gifu University \\ 1-1, Yanagido, Gifu, 501-1193 \\ Email: yamawaki@kmb.info.gifu-u.ac.jp, kamabe@ieee.org, shan.lu.jp@ieee.org
}

\begin{abstract}
Random input/output (RIO) code is a coding scheme that enables reading of one logical page using a single read threshold in multilevel flash memory. The construction of RIO codes is equivalent to the construction of WOM codes. Parallel RIO (P-RIO) code is an RIO code that encodes all pages in parallel. In this paper, we utilize coset coding with Hamming codes in order to construct P-RIO codes. Coset coding is a technique that constructs WOM codes using linear binary codes. We leverage the information on the data of all pages to encode each page. Our constructed codes store more pages than RIO codes constructed via coset coding.
\end{abstract}

\section{INTRODUCTION}

Flash memory is the prevalent type of non-volatile memory in use today, and flash devices are employed in universal serial bus (USB) memory technology, solid state drives (SSD), and mobile applications. A flash memory consists of an array of cells, in which information bits are stored in the form of the amount of charge. In conventional flash memory, one cell stores a single bit and the information is read using a single read threshold. Recently, multilevel flash memory technology has been introduced. In multilevel flash memory, each cell can represent one of more than two levels, and these levels are distinguished by multiple read thresholds. We consider the triple-level cell (TLC) that is currently being utilized in multilevel flash memory. A TLC can represent one of eight levels, that is, it stores three bits. Each level corresponds to a three-bit sequence as shown in Table I and each bit represents one logical page. Then, a group of such cells stores three

TABLE I

TRIPLE-LEVEL CELL (TLC)

\begin{tabular}{cccc} 
Level & Page 1 & Page 2 & Page 3 \\
\hline 7 & 1 & 0 & 0 \\
\hline 6 & 1 & 0 & 1 \\
\hline 5 & 1 & 1 & 1 \\
\hline 4 & 1 & 1 & 0 \\
\hline 3 & 0 & 1 & 0 \\
\hline 2 & 0 & 1 & 1 \\
\hline 1 & 0 & 0 & 1 \\
\hline 0 & 0 & 0 & 0 \\
\hline
\end{tabular}

logical pages, referred to as pages $1-3$. To read pages $1-$ 3 , the required numbers of read thresholds are 1,2 , and 4 , respectively. Therefore, the average number of read thresholds is 2.33. As stated above, multiple read thresholds are required in order to read a single logical page in multilevel flash memory. However, use of a large number of read thresholds degrades the read performance of the flash memory device.

In order to solve this problem, the random input/output (RIO) code has been proposed by Sharon and Alrod [1]. This is a coding scheme in which one logical page can be read using a single read threshold. Further, Sharon and Alrod showed that the construction of the RIO code is equivalent to the construction of the WOM code [1].

However, a distinction exists between the RIO code and the WOM code. In the WOM code, the data are stored sequentially. Therefore, when the data is encoded, the subsequent data are unknown. On the other hand, in the RIO code, the data of all logical pages are stored simultaneously; thus, all the data are known in advance. Previously, Yaakobi and Motwani proposed the parallel RIO (P-RIO) code, in which the encoding of each page is performed in parallel [3]. These researchers demonstrated P-RIO codes having parameters for which WOM codes or RIO codes do not exist. In addition, they proposed an algorithm to construct a P-RIO code via a computer search. However, the complexity of this algorithm increases exponentially with the code length.

In this paper, P-RIO codes are constructed using coset coding. Coset coding, introduced by Cohen, Godlewski, and Merkx, is a technique that constructs WOM codes using linear binary codes [4]. When Hamming codes are used as the linear codes, we leverage the information on the data of all logical pages to construct the P-RIO codes. Our constructed codes store more pages than RIO codes constructed via coset coding with Hamming codes. The remainder of the paper is structured as follows. In section $\amalg$, some preliminary notation and definitions are presented, whereas the construction of PRIO codes using coset coding is demonstrated in section III. Section IV contains a brief concluding section.

\section{PRELIMINARIES}

We first present some preliminary definitions and notation. For a positive integer $n$, we define $\mathcal{I}_{n}=\{1, \ldots, n\}$. In addition, for two vectors $\boldsymbol{x}=\left(x_{1}, \ldots, x_{n}\right)$ and $\boldsymbol{y}=\left(y_{1}, \ldots, y_{n}\right)$, we denote $\boldsymbol{x} \leq \boldsymbol{y}$ if $x_{i} \leq y_{i}$ for any $i \in \mathcal{I}_{n}$. For a binary vector $\boldsymbol{x}=\left(x_{1}, \ldots, x_{n}\right)$, we define $I(\boldsymbol{x})=\left\{i \mid i \in \mathcal{I}_{n}, x_{i}=1\right\}$. 
TABLE II

$[3,2,2]$ WOM CODE

\begin{tabular}{ccc} 
Data bits & First write & Second write \\
\hline 00 & 000 & 111 \\
\hline 01 & 100 & 011 \\
\hline 10 & 010 & 101 \\
\hline 11 & 001 & 110 \\
\hline
\end{tabular}

\section{A. WOM Code}

In a WOM, a cell stores a single bit and its content can be changed from 0 to 1 ; however, it cannot be changed from 1 to 0 . Rivest and Shamir have proposed a WOM code that allows multiple writings into WOM [2].

Definition 1: An $[n, l, t]$ WOM code is a coding scheme that permits the writing of $l$ data bits into $n$ cells $t$ times. This scheme is defined by the encoding map $\mathcal{E}$ and the decoding map $\mathcal{D}$. The former is defined by

$$
\mathcal{E}:\{0,1\}^{l} \times \operatorname{Im}\left(\mathcal{E}^{i-1}\right) \rightarrow\{0,1\}^{n},
$$

where $\operatorname{Im}\left(\mathcal{E}^{0}\right)=\{(0, \ldots, 0)\}$. For all $(\boldsymbol{d}, \boldsymbol{c}) \in\{0,1\}^{l} \times$ $\operatorname{Im}\left(\mathcal{E}^{i-1}\right)$ with $i \in \mathcal{I}_{t}, \boldsymbol{c} \leq \mathcal{E}(\boldsymbol{d}, \boldsymbol{c})$ is satisfied. Further, $\mathcal{D}$ is defined by

$$
\mathcal{D}: \operatorname{Im}\left(\mathcal{E}^{i}\right) \rightarrow\{0,1\}^{l},
$$

such that $\mathcal{D}(\mathcal{E}(\boldsymbol{d}, \boldsymbol{c}))=\boldsymbol{d}$ for all $(\boldsymbol{d}, \boldsymbol{c}) \in\{0,1\}^{l} \times \operatorname{Im}\left(\mathcal{E}^{i-1}\right)$ with $i \in \mathcal{I}_{t}$. The sum-rate is defined as $l t / n$.

Example 1: Previously, Rivest and Shamir presented the $[3,2,2]$ WOM code [2], which is shown in Table [I]

\section{B. Coset Coding}

In this paper, a linear binary code of length $n$ and dimension $k$ is referred to as an $(n, k)$ code. Coset coding is used to construct an $[n, n-k, t]$ WOM code using $(n, k)$ code $C$, where $t$ depends on $C$ [4]. Let $H$ be the parity check matrix of $C$. For all $(\boldsymbol{d}, \boldsymbol{c}) \in\{0,1\}^{n-k} \times \operatorname{Im}\left(\mathcal{E}^{i-1}\right)$ with $i \in \mathcal{I}_{t}$, the encoding map $\mathcal{E}$ is as follows.

$$
\mathcal{E}(\boldsymbol{d}, \boldsymbol{c})=\boldsymbol{c}+\boldsymbol{x},
$$

where

$$
\boldsymbol{x} \in\left\{\boldsymbol{v} \mid \boldsymbol{v} \in\{0,1\}^{n}, \boldsymbol{v} H^{T}=\boldsymbol{d}-\boldsymbol{c} H^{T}, I(\boldsymbol{c}) \cap I(\boldsymbol{v})=\emptyset\right\} .
$$

Further, for all $c \in \operatorname{Im}\left(\mathcal{E}^{i}\right)$ with $i \in \mathcal{I}_{t}$, the decoding map $\mathcal{D}$ is

$$
\mathcal{D}(\boldsymbol{c})=\boldsymbol{c} H^{T} .
$$

The following theorem has been proven in [4].

Theorem 1: When the linear code is the $(7,4)$ Hamming code, coset coding can be used to construct the $[7,3,3]$ WOM code. Let $r$ be an integer greater than 3 . Then a $\left[2^{r}-1, r, 2^{r-2}+2\right]$ WOM code can be constructed via coset coding with a $\left(2^{r}-1,2^{r}-r-1\right)$ Hamming code.
TABLE III

$[3,2,2]$ RIO CODE

\begin{tabular}{c|cccc} 
Data of page 2 & \multicolumn{4}{|c}{ Data of page 1 } \\
\cline { 2 - 5 } & 00 & 01 & 10 & 11 \\
\hline 00 & 000 & 211 & 121 & 112 \\
\hline 01 & 100 & 200 & 021 & 012 \\
\hline 10 & 010 & 201 & 020 & 102 \\
\hline 11 & 001 & 210 & 120 & 002 \\
\hline
\end{tabular}

\section{RIO Code}

In this paper, it is assumed that each cell of a flash memory represents one of $q$ levels $(0,1, \ldots, q-1)$. These levels are distinguished by $(q-1)$ different read thresholds. For each $i \in \mathcal{I}_{q-1}$, we denote the threshold between levels $(i-1)$ and $i$ by $i$. For the state of $n$ cells $\boldsymbol{c}=\left(c_{1}, \ldots, c_{n}\right) \in$ $\{0, \ldots, q-1\}^{n}$, the operation for read threshold $i$ is denoted by $R T_{i}(\boldsymbol{c})$. We define $R T_{i}(\boldsymbol{c})=\left(r_{1}, \ldots, r_{n}\right) \in\{0,1\}^{n}$ where, for each $j \in \mathcal{I}_{n}, r_{j}=1$ if $c_{j} \geq i$; otherwise, $r_{j}=0$. For any $\boldsymbol{c}_{1}, \ldots, \boldsymbol{c}_{q-1} \in\{0,1\}^{n}$ such that $\boldsymbol{c}_{1} \leq \boldsymbol{c}_{2} \leq \ldots \leq \boldsymbol{c}_{q-1}$, the following property holds: For each $i \in \mathcal{I}_{q-1}$,

$$
R T_{q-i}\left(\sum_{j=1}^{q-1} \boldsymbol{c}_{j}\right)=\boldsymbol{c}_{i} .
$$

RIO code is a coding scheme that stores $t$ pages in a $(t+1)$ level flash memory, such that page $i$ can be read using read threshold $(t+1-i)$ for each $i \in \mathcal{I}_{t}$ [1]. From (6), pages $1, \ldots, t$ are encoded into $\boldsymbol{c}_{1}, \ldots, \boldsymbol{c}_{t}$, respectively, such that $\boldsymbol{c}_{1} \leq \boldsymbol{c}_{2} \leq \cdots \leq \boldsymbol{c}_{t}$, and the cell state is set to $\sum_{i=1}^{t} \boldsymbol{c}_{i}$, where $\boldsymbol{c}_{i}$ is a binary vector for each $i \in \mathcal{I}_{t}$.

Definition 2: An $[n, l, t]$ RIO code is a coding scheme that enables storage of $t$ pages of $l$ data bits in $n(t+1)$-level cells, such that each page can be read using a single read threshold. For each $i \in \mathcal{I}_{t}$, the encoding map of page $i \mathcal{E}_{i}$ is defined by

$$
\mathcal{E}_{i}:\{0,1\}^{l} \times \operatorname{Im}\left(\mathcal{E}_{i-1}\right) \rightarrow\{0,1\}^{n},
$$

where $\operatorname{Im}\left(\mathcal{E}_{0}\right)=\{(0, \ldots, 0)\}$. For all $(\boldsymbol{d}, \boldsymbol{c}) \in\{0,1\}^{l} \times$ $\operatorname{Im}\left(\mathcal{E}_{i-1}\right), \boldsymbol{c} \leq \mathcal{E}_{i}(\boldsymbol{d}, \boldsymbol{c})$ is satisfied. The cell state is the sum of the $\mathcal{E}_{i}$ results for all $i \in \mathcal{I}_{t}$. Further, for each $i \in \mathcal{I}_{t}$, the decoding map of page $i \mathcal{D}_{i}$ is defined by

$$
\mathcal{D}_{i}: \operatorname{Im}\left(\mathcal{E}_{i}\right) \rightarrow\{0,1\}^{l},
$$

such that $\mathcal{D}_{i}\left(\mathcal{E}_{i}(\boldsymbol{d}, \boldsymbol{c})\right)=\boldsymbol{d}$ for all $(\boldsymbol{d}, \boldsymbol{c}) \in\{0,1\}^{l} \times \operatorname{Im}\left(\mathcal{E}_{i-1}\right)$. If the cell state is $c \in\{0, \ldots, t\}^{n}$, the argument of $\mathcal{D}_{i}$ is $R T_{t+1-i}(\boldsymbol{c})$ for each $i \in \mathcal{I}_{t}$.

From Definitions 1 and 2, it is apparent that the construction of an $[n, l, t]$ RIO code is equivalent to that of an $[n, l, t]$ WOM code [1].

Example 2: The $[3,2,2]$ RIO code based on the $[3,2,2]$ WOM code is shown in Table III. As an example, let the data of pages 1 and 2 be 10 and 01, respectively. Then, from Table [II. $\mathcal{E}_{1}(10,000)=010$ and $\mathcal{E}_{2}(01,010)=011$. Therefore, the cell state is 021 . 


\section{P-RIO Code}

In the RIO code, each page encoding depends on the page data only. In contrast, for P-RIO code, information on the data of all pages is leveraged during encoding of each page [3].

Definition 3: An $[n, l, t]$ P-RIO code is an $[n, l, t]$ RIO code for which all pages are encoded in parallel. The encoding map $\mathcal{E}$ is defined by

$$
\mathcal{E}: \prod_{i=1}^{t}\{0,1\}^{l} \rightarrow \prod_{i=1}^{t}\{0,1\}^{n} .
$$

For all $\left(\boldsymbol{d}_{1}, \ldots, \boldsymbol{d}_{t}\right) \in \prod_{i=1}^{t}\{0,1\}^{l}, \boldsymbol{c}_{1} \leq \boldsymbol{c}_{2} \leq \cdots \leq \boldsymbol{c}_{t}$ is satisfied, where $\left(\boldsymbol{c}_{1}, \ldots, \boldsymbol{c}_{t}\right)=\mathcal{E}\left(\boldsymbol{d}_{1}, \ldots, \boldsymbol{d}_{t}\right)$. The cell state is the sum of the components of the $\mathcal{E}$ result. For each $i \in \mathcal{I}_{t}$, the decoding map $\mathcal{D}_{i}$ of page $i$ is defined by

$$
\mathcal{D}_{i}:\{0,1\}^{n} \rightarrow\{0,1\}^{l},
$$

such that $\mathcal{D}_{i}\left(\boldsymbol{c}_{i}\right)=\boldsymbol{d}_{i}$ for all $\left(\boldsymbol{d}_{1}, \ldots, \boldsymbol{d}_{t}\right) \in \prod_{i=1}^{t}\{0,1\}^{l}$, where $\left(\boldsymbol{c}_{1}, \ldots, \boldsymbol{c}_{t}\right)=\mathcal{E}\left(\boldsymbol{d}_{1}, \ldots, \boldsymbol{d}_{t}\right)$. If the cell state is $\boldsymbol{c} \in$ $\{0, \ldots, t\}^{n}$, the argument of $\mathcal{D}_{i}$ is $R T_{t+1-i}(\boldsymbol{c})$ for each $i \in \mathcal{I}_{t}$.

An algorithm to construct P-RIO code has been proposed in a previous study [3], and run to yield P-RIO codes that store two pages for moderate code lengths. These codes have parameters for which RIO codes do not exist [3].

In this study, we use coset coding to construct P-RIO codes. When Hamming codes are used as the linear codes, from Theorem 1, [7,3,3] RIO code and $[15,4,6]$ RIO code can be constructed. Then, we leverage the information on the data of all pages to construct P-RIO codes that store more pages than these RIO codes.

\section{Construction of P-RiO Code using Coset CODING}

Prior to construction of P-RIO codes using coset coding, we first discuss several properties.

\section{A. Properties}

We have the following theorem.

Theorem 2: Let $H$ be the parity check matrix of $(n, k)$ code $C$. The sufficient condition that uses coset coding with $C$ to construct an $[n, n-k, t] \mathrm{P}-\mathrm{RIO}$ code is as follows. For any $\boldsymbol{s}_{1}, \ldots, \boldsymbol{s}_{t} \in\{0,1\}^{n-k}$, there exist $\boldsymbol{x}_{1}, \ldots, \boldsymbol{x}_{t} \in\{0,1\}^{n}$ that satisfy the following conditions:

1) For all $i \in \mathcal{I}_{t}, \boldsymbol{x}_{i} H^{T}=\boldsymbol{s}_{i}$;

2) For all $i, i^{\prime} \in \mathcal{I}_{t}, i \neq i^{\prime}, I\left(\boldsymbol{x}_{i}\right) \cap I\left(\boldsymbol{x}_{i^{\prime}}\right)=\emptyset$.

Proof: For any $\boldsymbol{d}_{1}, \ldots, \boldsymbol{d}_{t} \in\{0,1\}^{n-k}$, we define $\boldsymbol{s}_{i}=$ $\boldsymbol{d}_{i}-\boldsymbol{d}_{i-1}$ for each $i \in \mathcal{I}_{t}$, where $\boldsymbol{d}_{0}=(0, \ldots, 0)$. Suppose that there exist $\boldsymbol{x}_{1}, \ldots, \boldsymbol{x}_{t} \in\{0,1\}^{n}$ that satisfy the above conditions for $\boldsymbol{s}_{1}, \ldots, \boldsymbol{s}_{t}$. We define $\boldsymbol{c}_{i}=\sum_{i^{\prime}=1}^{i} \boldsymbol{x}_{i^{\prime}}$ for each $i \in \mathcal{I}_{t}$. Then, we have $\boldsymbol{c}_{i} H^{T}=\boldsymbol{d}_{i}$ for each $i \in \mathcal{I}_{t}$ and $\boldsymbol{c}_{1} \leq \boldsymbol{c}_{2} \leq \cdots \leq \boldsymbol{c}_{t}$. Therefore, an $[n, n-k, t]$ P-RIO code can be constructed, where $\mathcal{E}\left(\boldsymbol{d}_{1}, \ldots, \boldsymbol{d}_{t}\right)=\left(\boldsymbol{c}_{1}, \ldots, \boldsymbol{c}_{t}\right)$ and $\mathcal{D}_{i}\left(\boldsymbol{c}_{i}\right)=\boldsymbol{c}_{i} H^{T}$.

For $r \geq 3$, we denote the parity check matrix of the $\left(2^{r}-\right.$ $\left.1,2^{r}-r-1\right)$ Hamming code by

$$
H_{r}=\left(\begin{array}{llll}
\boldsymbol{h}_{1}^{T} & \boldsymbol{h}_{2}^{T} & \cdots & \boldsymbol{h}_{2^{r}-1}^{T}
\end{array}\right),
$$

where $\left\{\boldsymbol{h}_{1}, \boldsymbol{h}_{2}, \ldots, \boldsymbol{h}_{2^{r}-1}\right\}=\{0,1\}^{r} \backslash\{(0, \ldots, 0)\}$. For $\boldsymbol{s} \in$ $\{0,1\}^{r}$, we define $V(\boldsymbol{s})=\left\{\{j\} \mid j \in \mathcal{I}_{2^{r}-1}, \boldsymbol{h}_{j}=\boldsymbol{s}\right\} \cup$ $\left\{\left\{j_{1}, j_{2}\right\} \mid j_{1}, j_{2} \in \mathcal{I}_{2^{r}-1}, \boldsymbol{h}_{j_{1}}+\boldsymbol{h}_{j_{2}}=\boldsymbol{s}\right\}$. Then, the following theorem holds.

Theorem 3: For any $s \in\{0,1\}^{r} \backslash\{(0, \ldots, 0)\}$, we have the permutation $\sigma$ of $\mathcal{I}_{2^{r}-1}$ such that

$$
\begin{array}{r}
V(\boldsymbol{s})=\{\{\sigma(1)\},\{\sigma(2), \\
\sigma(3)\},\{\sigma(4), \sigma(5)\}, \ldots, \\
\left.\left\{\sigma\left(2^{r}-2\right), \sigma\left(2^{r}-1\right)\right\}\right\} .
\end{array}
$$

Proof: Let $\alpha_{1}$ be an integer such that $\alpha_{1} \in \mathcal{I}_{2^{r}-1}$ and

$$
\boldsymbol{s}=\boldsymbol{h}_{\alpha_{1}} .
$$

Then, $\left\{\alpha_{1}\right\} \in V(s)$. Further, let $\alpha_{2}$ be an integer such that $\alpha_{2} \in \mathcal{I}_{2^{r}-1} \backslash\left\{\alpha_{1}\right\}$, and let $\alpha_{3}$ be an integer such that $\alpha_{3} \in$ $\mathcal{I}_{2^{r}-1} \backslash\left\{\alpha_{1}, \alpha_{2}\right\}$ and

$$
\boldsymbol{s}=\boldsymbol{h}_{\alpha_{2}}+\boldsymbol{h}_{\alpha_{3}} \text {. }
$$

Then, $\left\{\alpha_{2}, \alpha_{3}\right\} \in V(s)$. As with $\alpha_{2}$ and $\alpha_{3}$, we have $\alpha_{4}, \alpha_{5}, \ldots, \alpha_{2^{r}-2}, \alpha_{2^{r}-1}$. Then, $\left\{\alpha_{4}, \alpha_{5}\right\}, \ldots,\left\{\alpha_{2^{r}-2}, \alpha_{2^{r}-1}\right\} \in V(s)$. Let $\sigma$ be the permutation of $\mathcal{I}_{2^{r}-1}$ such that $\sigma(j)=\alpha_{j}$ for each $j \in \mathcal{I}_{2^{r}-1}$. Then, $\sigma$ satisfies (12).

Theorem 4: For any $\boldsymbol{s}_{1}, \boldsymbol{s}_{2} \in\{0,1\}^{r} \backslash\{(0, \ldots, 0)\}, \boldsymbol{s}_{1} \neq \boldsymbol{s}_{2}$, we have the permutation $\sigma$ of $\mathcal{I}_{2^{r}-1}$ such that

$$
\begin{aligned}
& V\left(s_{1}\right) \\
&=\quad\{\{\sigma(1)\},\{\sigma(2), \sigma(3)\},\{\sigma(4), \sigma(5)\},\{\sigma(6), \sigma(7)\}, \\
&\left.\ldots,\left\{\sigma\left(2^{r}-4\right), \sigma\left(2^{r}-3\right)\right\},\left\{\sigma\left(2^{r}-2\right), \sigma\left(2^{r}-1\right)\right\}\right\}, \\
& V\left(s_{2}\right) \\
&=\quad\{\{\sigma(2)\},\{\sigma(1), \sigma(3)\},\{\sigma(4), \sigma(6)\},\{\sigma(5), \sigma(7)\}, \\
&\left.\ldots,\left\{\sigma\left(2^{r}-4\right), \sigma\left(2^{r}-2\right)\right\},\left\{\sigma\left(2^{r}-3\right), \sigma\left(2^{r}-1\right)\right\}\right\} .
\end{aligned}
$$

Proof: Let $\alpha_{1}$ be an integer such that $\alpha_{1} \in \mathcal{I}_{2^{r}-1}$ and

$$
\boldsymbol{s}_{1}=\boldsymbol{h}_{\alpha_{1}} \text {. }
$$

Let $\alpha_{2}$ be an integer such that $\alpha_{2} \in \mathcal{I}_{2^{r}-1}$ and

$$
\boldsymbol{s}_{2}=\boldsymbol{h}_{\alpha_{2}} \text {. }
$$

Then, $\left\{\alpha_{1}\right\} \in V\left(s_{1}\right),\left\{\alpha_{2}\right\} \in V\left(s_{2}\right)$. Clearly, $\alpha_{1} \neq \alpha_{2}$. Let $\alpha_{3}$ be an integer such that $\alpha_{3} \in \mathcal{I}_{2^{r}-1} \backslash\left\{\alpha_{1}, \alpha_{2}\right\}$ and

$$
\boldsymbol{s}_{1}=\boldsymbol{h}_{\alpha_{2}}+\boldsymbol{h}_{\alpha_{3}} \text {. }
$$

Then, $\left\{\alpha_{2}, \alpha_{3}\right\} \in V\left(s_{1}\right)$. From (17), (19), and (18),

$$
\begin{aligned}
\boldsymbol{h}_{\alpha_{1}}+\boldsymbol{h}_{\alpha_{3}} & =\boldsymbol{s}_{1}+\boldsymbol{h}_{\alpha_{3}}=\left(\boldsymbol{h}_{\alpha_{2}}+\boldsymbol{h}_{\alpha_{3}}\right)+\boldsymbol{h}_{\alpha_{3}} \\
& =\boldsymbol{h}_{\alpha_{2}}=\boldsymbol{s}_{2} .
\end{aligned}
$$

Hence, $\left\{\alpha_{1}, \alpha_{3}\right\} \in V\left(s_{2}\right)$. Let $\alpha_{4}$ be an integer such that $\alpha_{4} \in \mathcal{I}_{2^{r}-1} \backslash\left\{\alpha_{1}, \ldots, \alpha_{3}\right\}$, and let $\alpha_{5}$ be an integer such that $\alpha_{5} \in \mathcal{I}_{2^{r}-1} \backslash\left\{\alpha_{1}, \ldots, \alpha_{4}\right\}$ and

$$
\boldsymbol{s}_{1}=\boldsymbol{h}_{\alpha_{4}}+\boldsymbol{h}_{\alpha_{5}} \text {. }
$$


Then, $\left\{\alpha_{4}, \alpha_{5}\right\} \in V\left(s_{1}\right)$. Let $\alpha_{6}$ be an integer such that $\alpha_{6} \in$ $\mathcal{I}_{2^{r}-1} \backslash\left\{\alpha_{1}, \ldots, \alpha_{4}\right\}$ and

$$
\boldsymbol{s}_{2}=\boldsymbol{h}_{\alpha_{4}}+\boldsymbol{h}_{\alpha_{6}} .
$$

Then, $\left\{\alpha_{4}, \alpha_{6}\right\} \in V\left(s_{2}\right)$. Clearly, $\alpha_{5} \neq \alpha_{6}$. Let $\alpha_{7}$ be an integer such that $\alpha_{7} \in \mathcal{I}_{2^{r}-1} \backslash\left\{\alpha_{1}, \ldots, \alpha_{6}\right\}$ and

$$
\boldsymbol{s}_{1}=\boldsymbol{h}_{\alpha_{6}}+\boldsymbol{h}_{\alpha_{7}} \text {. }
$$

Then, $\left\{\alpha_{6}, \alpha_{7}\right\} \in V\left(s_{1}\right)$. From (21) and (23),

$$
\boldsymbol{h}_{\alpha_{4}}+\boldsymbol{h}_{\alpha_{5}}=\boldsymbol{h}_{\alpha_{6}}+\boldsymbol{h}_{\alpha_{7}} .
$$

From (24) and (22),

$$
\boldsymbol{h}_{\alpha_{5}}+\boldsymbol{h}_{\alpha_{7}}=\boldsymbol{h}_{\alpha_{4}}+\boldsymbol{h}_{\alpha_{6}}=\boldsymbol{s}_{2} \text {. }
$$

Hence, $\left\{\alpha_{5}, \alpha_{7}\right\} \in V\left(s_{2}\right)$. As with $\alpha_{4}, \alpha_{5}, \alpha_{6}, \alpha_{7}$, we have $\alpha_{8}, \alpha_{9}, \alpha_{10}, \alpha_{11}, \ldots, \alpha_{2^{r}-4}, \alpha_{2^{r}-3}, \alpha_{2^{r}-2}, \alpha_{2^{r}-1}$. Let $\sigma$ be the permutation of $\mathcal{I}_{2^{r}-1}$ such that $\sigma(j)=\alpha_{j}$ for each $j \in \mathcal{I}_{2^{r}-1}$. Then, $\sigma$ satisfies (15) and (16).

Theorem 5: Let $r=4$. For any $\boldsymbol{s}_{1}, \boldsymbol{s}_{2}, \boldsymbol{s}_{3} \in\{0,1\}^{4} \backslash$ $\{0000\}\left(s_{i} \neq s_{i^{\prime}}\right.$ for any $\left.i, i^{\prime} \in \mathcal{I}_{3}, i \neq i^{\prime}\right)$, we have the permutation $\sigma$ of $\mathcal{I}_{15}$, which satisfies the following conditions: (a) If $s_{1}+s_{2} \neq s_{3}$,

$$
\begin{aligned}
V\left(\boldsymbol{s}_{1}\right)= & \{\{\sigma(1)\},\{\sigma(2), \sigma(3)\},\{\sigma(4), \sigma(5)\}, \\
& \{\sigma(6), \sigma(7)\},\{\sigma(8), \sigma(9)\},\{\sigma(10), \sigma(11)\}, \\
& \{\sigma(12), \sigma(13)\},\{\sigma(14), \sigma(15)\}\}, \\
V\left(s_{2}\right)= & \{\{\sigma(2)\},\{\sigma(1), \sigma(3)\},\{\sigma(4), \sigma(6)\}, \\
& \{\sigma(5), \sigma(7)\},\{\sigma(8), \sigma(10)\},\{\sigma(9), \sigma(11)\}, \\
& \{\sigma(12), \sigma(14)\},\{\sigma(13), \sigma(15)\}\}, \\
V\left(s_{3}\right)= & \{\{\sigma(4)\},\{\sigma(1), \sigma(5)\},\{\sigma(2), \sigma(6)\}, \\
& \{\sigma(3), \sigma(7)\},\{\sigma(8), \sigma(12)\},\{\sigma(9), \sigma(13)\}, \\
& \{\sigma(10), \sigma(14)\},\{\sigma(11), \sigma(15)\}\} .
\end{aligned}
$$

(b) If $s_{1}+s_{2}=s_{3}$,

$$
\begin{aligned}
V\left(s_{1}\right)= & \{\{\sigma(1)\},\{\sigma(2), \sigma(3)\},\{\sigma(4), \sigma(5)\}, \\
& \{\sigma(6), \sigma(7)\},\{\sigma(8), \sigma(9)\},\{\sigma(10), \sigma(11)\}, \\
& \{\sigma(12), \sigma(13)\},\{\sigma(14), \sigma(15)\}\}, \\
V\left(s_{2}\right)= & \{\{\sigma(2)\},\{\sigma(1), \sigma(3)\},\{\sigma(4), \sigma(6)\}, \\
& \{\sigma(5), \sigma(7)\},\{\sigma(8), \sigma(10)\},\{\sigma(9), \sigma(11)\}, \\
& \{\sigma(12), \sigma(14)\},\{\sigma(13), \sigma(15)\}\}, \\
V\left(s_{3}\right)= & \{\{\sigma(3)\},\{\sigma(1), \sigma(2)\},\{\sigma(4), \sigma(7)\}, \\
& \{\sigma(5), \sigma(6)\},\{\sigma(8), \sigma(11)\},\{\sigma(9), \sigma(10)\}, \\
& \{\sigma(12), \sigma(15)\},\{\sigma(13), \sigma(14)\}\} .
\end{aligned}
$$

Proof: Let $\alpha_{1}$ be an integer such that $\alpha_{1} \in \mathcal{I}_{15}$ and

$$
\boldsymbol{s}_{1}=\boldsymbol{h}_{\alpha_{1}} \text {. }
$$

Let $\alpha_{2}$ be an integer such that $\alpha_{2} \in \mathcal{I}_{15}$ and

$$
\boldsymbol{s}_{2}=\boldsymbol{h}_{\alpha_{2}} \text {. }
$$

Then, $\left\{\alpha_{1}\right\} \in V\left(s_{1}\right),\left\{\alpha_{2}\right\} \in V\left(s_{2}\right)$. Clearly, $\alpha_{1} \neq \alpha_{2}$. Let $\alpha_{3}$ be an integer such that $\alpha_{3} \in \mathcal{I}_{15} \backslash\left\{\alpha_{1}, \alpha_{2}\right\}$ and

$$
\boldsymbol{s}_{1}=\boldsymbol{h}_{\alpha_{2}}+\boldsymbol{h}_{\alpha_{3}} .
$$

Then, $\left\{\alpha_{2}, \alpha_{3}\right\} \in V\left(s_{1}\right)$. From (32), (34), and (33),

$$
\begin{aligned}
\boldsymbol{h}_{\alpha_{1}}+\boldsymbol{h}_{\alpha_{3}} & =\boldsymbol{s}_{1}+\boldsymbol{h}_{\alpha_{3}}=\left(\boldsymbol{h}_{\alpha_{2}}+\boldsymbol{h}_{\alpha_{3}}\right)+\boldsymbol{h}_{\alpha_{3}} \\
& =\boldsymbol{h}_{\alpha_{2}}=\boldsymbol{s}_{2} .
\end{aligned}
$$

Hence, $\left\{\alpha_{1}, \alpha_{3}\right\} \in V\left(s_{2}\right)$.

(a) Let $\alpha_{4}$ be an integer such that $\alpha_{4} \in \mathcal{I}_{15}$ and

$$
\boldsymbol{s}_{3}=\boldsymbol{h}_{\alpha_{4}} \text {. }
$$

Then, $\left\{\alpha_{4}\right\} \in V\left(s_{3}\right)$. Clearly, $\alpha_{4} \notin\left\{\alpha_{1}, \alpha_{2}\right\}$. From (36), (34), and (33),

$$
\boldsymbol{h}_{\alpha_{4}}=\boldsymbol{s}_{3} \neq \boldsymbol{s}_{1}+\boldsymbol{s}_{2}=\left(\boldsymbol{h}_{\alpha_{2}}+\boldsymbol{h}_{\alpha_{3}}\right)+\boldsymbol{h}_{\alpha_{2}}=\boldsymbol{h}_{\alpha_{3}} .
$$

Therefore, $\alpha_{4} \neq \alpha_{3}$. Let $\alpha_{5}$ be an integer such that $\alpha_{5} \in$ $\mathcal{I}_{15} \backslash\left\{\alpha_{1}, \ldots, \alpha_{4}\right\}$ and

$$
\boldsymbol{s}_{1}=\boldsymbol{h}_{\alpha_{4}}+\boldsymbol{h}_{\alpha_{5}} .
$$

Then, $\left\{\alpha_{4}, \alpha_{5}\right\} \in V\left(s_{1}\right)$. From (32) and (38),

$$
\boldsymbol{h}_{\alpha_{1}}=\boldsymbol{h}_{\alpha_{4}}+\boldsymbol{h}_{\alpha_{5}} .
$$

From (39) and 36,

$$
\boldsymbol{h}_{\alpha_{1}}+\boldsymbol{h}_{\alpha_{5}}=\boldsymbol{h}_{\alpha_{4}}=\boldsymbol{s}_{3} \text {. }
$$

Hence, $\left\{\alpha_{1}, \alpha_{5}\right\} \in V\left(s_{3}\right)$. Let $\alpha_{6}$ be an integer such that $\alpha_{6} \in \mathcal{I}_{15} \backslash\left\{\alpha_{1}, \ldots, \alpha_{5}\right\}$ and

$$
\boldsymbol{s}_{2}=\boldsymbol{h}_{\alpha_{4}}+\boldsymbol{h}_{\alpha_{6}} .
$$

Then $\left\{\alpha_{4}, \alpha_{6}\right\} \in V\left(s_{2}\right)$. From (33) and (41),

$$
\boldsymbol{h}_{\alpha_{2}}=\boldsymbol{h}_{\alpha_{4}}+\boldsymbol{h}_{\alpha_{6}} \text {. }
$$

From (42) and 36,

$$
\boldsymbol{h}_{\alpha_{2}}+\boldsymbol{h}_{\alpha_{6}}=\boldsymbol{h}_{\alpha_{4}}=\boldsymbol{s}_{3} \text {. }
$$

Therefore, $\left\{\alpha_{2}, \alpha_{6}\right\} \in V\left(s_{3}\right)$. Let $\alpha_{7}$ be an integer such that $\alpha_{7} \in \mathcal{I}_{15} \backslash\left\{\alpha_{1}, \ldots, \alpha_{6}\right\}$ and

$$
\boldsymbol{s}_{1}=\boldsymbol{h}_{\alpha_{6}}+\boldsymbol{h}_{\alpha_{7}} \text {. }
$$

Then, $\left\{\alpha_{6}, \alpha_{7}\right\} \in V\left(s_{1}\right)$. From (38) and (44),

$$
\boldsymbol{h}_{\alpha_{4}}+\boldsymbol{h}_{\alpha_{5}}=\boldsymbol{h}_{\alpha_{6}}+\boldsymbol{h}_{\alpha_{7}} .
$$

From (45) and (41),

$$
\boldsymbol{h}_{\alpha_{5}}+\boldsymbol{h}_{\alpha_{7}}=\boldsymbol{h}_{\alpha_{4}}+\boldsymbol{h}_{\alpha_{6}}=\boldsymbol{s}_{2} \text {. }
$$

Hence, $\left\{\alpha_{5}, \alpha_{7}\right\} \in V\left(s_{2}\right)$. From (34) and (44),

$$
\boldsymbol{h}_{\alpha_{2}}+\boldsymbol{h}_{\alpha_{3}}=\boldsymbol{h}_{\alpha_{6}}+\boldsymbol{h}_{\alpha_{7}} .
$$

From (47) and 43,

$$
\boldsymbol{h}_{\alpha_{3}}+\boldsymbol{h}_{\alpha_{7}}=\boldsymbol{h}_{\alpha_{2}}+\boldsymbol{h}_{\alpha_{6}}=\boldsymbol{s}_{3} .
$$


Therefore, $\left\{\alpha_{3}, \alpha_{7}\right\} \in V\left(s_{3}\right)$. Let $\alpha_{8}$ be an integer such that $\alpha_{8} \in \mathcal{I}_{15} \backslash\left\{\alpha_{1}, \ldots, \alpha_{7}\right\}$, and let $\alpha_{9}$ be an integer such that $\alpha_{9} \in \mathcal{I}_{15} \backslash\left\{\alpha_{1}, \ldots, \alpha_{8}\right\}$ and

$$
\boldsymbol{s}_{1}=\boldsymbol{h}_{\alpha_{8}}+\boldsymbol{h}_{\alpha_{9}} \text {. }
$$

Then, $\left\{\alpha_{8}, \alpha_{9}\right\} \in V\left(s_{1}\right)$. Let $\alpha_{10}$ be an integer such that $\alpha_{10} \in \mathcal{I}_{15} \backslash\left\{\alpha_{1}, \ldots, \alpha_{9}\right\}$ and

$$
\boldsymbol{s}_{2}=\boldsymbol{h}_{\alpha_{8}}+\boldsymbol{h}_{\alpha_{10}} .
$$

Then, $\left\{\alpha_{8}, \alpha_{10}\right\} \in V\left(\boldsymbol{s}_{2}\right)$. Let $\alpha_{11}$ be an integer such that $\alpha_{11} \in \mathcal{I}_{15} \backslash\left\{\alpha_{1}, \ldots, \alpha_{10}\right\}$ and

$$
\boldsymbol{s}_{1}=\boldsymbol{h}_{\alpha_{10}}+\boldsymbol{h}_{\alpha_{11}} \text {. }
$$

Then, $\left\{\alpha_{10}, \alpha_{11}\right\} \in V\left(s_{1}\right)$. From (49) and (51),

$$
\boldsymbol{h}_{\alpha_{8}}+\boldsymbol{h}_{\alpha_{9}}=\boldsymbol{h}_{\alpha_{10}}+\boldsymbol{h}_{\alpha_{11}} \text {. }
$$

From (52) and (50),

$$
\boldsymbol{h}_{\alpha_{9}}+\boldsymbol{h}_{\alpha_{11}}=\boldsymbol{h}_{\alpha_{8}}+\boldsymbol{h}_{\alpha_{10}}=\boldsymbol{s}_{2} .
$$

Hence, $\left\{\alpha_{9}, \alpha_{11}\right\} \in V\left(s_{2}\right)$. Let $\alpha_{12}$ be an integer such that $\alpha_{12} \in \mathcal{I}_{15} \backslash\left\{\alpha_{1}, \ldots, \alpha_{10}\right\}$ and

$$
\boldsymbol{s}_{3}=\boldsymbol{h}_{\alpha_{8}}+\boldsymbol{h}_{\alpha_{12}} .
$$

Then, $\left\{\alpha_{8}, \alpha_{12}\right\} \in V\left(s_{3}\right)$. From (49) and (53),

$\boldsymbol{s}_{1}+\boldsymbol{s}_{2}=\left(\boldsymbol{h}_{\alpha_{8}}+\boldsymbol{h}_{\alpha_{9}}\right)+\left(\boldsymbol{h}_{\alpha_{9}}+\boldsymbol{h}_{\alpha_{11}}\right)=\boldsymbol{h}_{\alpha_{8}}+\boldsymbol{h}_{\alpha_{11}}$.

From (54) and (55),

$$
\boldsymbol{h}_{\alpha_{8}}+\boldsymbol{h}_{\alpha_{12}}=\boldsymbol{s}_{3} \neq \boldsymbol{s}_{1}+\boldsymbol{s}_{2}=\boldsymbol{h}_{\alpha_{8}}+\boldsymbol{h}_{\alpha_{11}} .
$$

Therefore, $\alpha_{12} \neq \alpha_{11}$. Let $\alpha_{13}$ be an integer such that $\alpha_{13} \in$ $\mathcal{I}_{15} \backslash\left\{\alpha_{1}, \ldots, \alpha_{12}\right\}$ and

$$
\boldsymbol{s}_{1}=\boldsymbol{h}_{\alpha_{12}}+\boldsymbol{h}_{\alpha_{13}} .
$$

Then, $\left\{\alpha_{12}, \alpha_{13}\right\} \in V\left(s_{1}\right)$. From (49) and (57),

$$
\boldsymbol{h}_{\alpha_{8}}+\boldsymbol{h}_{\alpha_{9}}=\boldsymbol{h}_{\alpha_{12}}+\boldsymbol{h}_{\alpha_{13}} \text {. }
$$

From (58) and (54),

$$
\boldsymbol{h}_{\alpha_{9}}+\boldsymbol{h}_{\alpha_{13}}=\boldsymbol{h}_{\alpha_{8}}+\boldsymbol{h}_{\alpha_{12}}=\boldsymbol{s}_{3} .
$$

Hence, $\left\{\alpha_{9}, \alpha_{13}\right\} \in V\left(s_{3}\right)$. Let $\alpha_{14}$ be an integer such that $\alpha_{14} \in \mathcal{I}_{15} \backslash\left\{\alpha_{1}, \ldots, \alpha_{13}\right\}$ and

$$
\boldsymbol{s}_{2}=\boldsymbol{h}_{\alpha_{12}}+\boldsymbol{h}_{\alpha_{14}} .
$$

Then $\left\{\alpha_{12}, \alpha_{14}\right\} \in V\left(s_{2}\right)$. From (50) and (60),

$$
\boldsymbol{h}_{\alpha_{8}}+\boldsymbol{h}_{\alpha_{10}}=\boldsymbol{h}_{\alpha_{12}}+\boldsymbol{h}_{\alpha_{14}} .
$$

From (61) and (54),

$$
\boldsymbol{h}_{\alpha_{10}}+\boldsymbol{h}_{\alpha_{14}}=\boldsymbol{h}_{\alpha_{8}}+\boldsymbol{h}_{\alpha_{12}}=\boldsymbol{s}_{3} .
$$

Therefore, $\left\{\alpha_{10}, \alpha_{14}\right\} \in V\left(s_{3}\right)$. Let $\alpha_{15}$ be an integer such that $\alpha_{15} \in \mathcal{I}_{15} \backslash\left\{\alpha_{1}, \ldots, \alpha_{14}\right\}$ and

$$
\boldsymbol{s}_{1}=\boldsymbol{h}_{\alpha_{14}}+\boldsymbol{h}_{\alpha_{15}} \text {. }
$$

Then, $\left\{\alpha_{14}, \alpha_{15}\right\} \in V\left(s_{1}\right)$. From (57) and (63),

$$
\boldsymbol{h}_{\alpha_{12}}+\boldsymbol{h}_{\alpha_{13}}=\boldsymbol{h}_{\alpha_{14}}+\boldsymbol{h}_{\alpha_{15}} .
$$

From (64) and 60),

$$
\boldsymbol{h}_{\alpha_{13}}+\boldsymbol{h}_{\alpha_{15}}=\boldsymbol{h}_{\alpha_{12}}+\boldsymbol{h}_{\alpha_{14}}=\boldsymbol{s}_{2} .
$$

Hence, $\left\{\alpha_{13}, \alpha_{15}\right\} \in V\left(s_{2}\right)$. From (51) and (63),

$$
\boldsymbol{h}_{\alpha_{10}}+\boldsymbol{h}_{\alpha_{11}}=\boldsymbol{h}_{\alpha_{14}}+\boldsymbol{h}_{\alpha_{15}} .
$$

From (66) and (62),

$$
\boldsymbol{h}_{\alpha_{11}}+\boldsymbol{h}_{\alpha_{15}}=\boldsymbol{h}_{\alpha_{10}}+\boldsymbol{h}_{\alpha_{14}}=\boldsymbol{s}_{3} .
$$

Therefore, $\left\{\alpha_{11}, \alpha_{15}\right\} \in V\left(s_{3}\right)$. Let $\sigma$ be the permutation of $\mathcal{I}_{15}$ such that $\sigma(j)=\alpha_{j}$ for each $j \in \mathcal{I}_{15}$. Then, $\sigma$ satisfies (26), (27), and (28).

(b) From (34) and (33),

$$
\boldsymbol{s}_{3}=\boldsymbol{s}_{1}+\boldsymbol{s}_{2}=\left(\boldsymbol{h}_{\alpha_{2}}+\boldsymbol{h}_{\alpha_{3}}\right)+\boldsymbol{h}_{\alpha_{2}}=\boldsymbol{h}_{\alpha_{3}} .
$$

Hence, $\left\{\alpha_{3}\right\} \in V\left(s_{3}\right)$. From (32) and (33),

$$
\boldsymbol{h}_{\alpha_{1}}+\boldsymbol{h}_{\alpha_{2}}=\boldsymbol{s}_{1}+\boldsymbol{s}_{2}=\boldsymbol{s}_{3} .
$$

Therefore, $\left\{\alpha_{1}, \alpha_{2}\right\} \in V\left(s_{3}\right)$. Let $\alpha_{4}$ be an integer such that $\alpha_{4} \in \mathcal{I}_{15} \backslash\left\{\alpha_{1}, \ldots, \alpha_{3}\right\}$, and let $\alpha_{5}$ be an integer such that $\alpha_{5} \in \mathcal{I}_{15} \backslash\left\{\alpha_{1}, \ldots, \alpha_{4}\right\}$ and

$$
\boldsymbol{s}_{1}=\boldsymbol{h}_{\alpha_{4}}+\boldsymbol{h}_{\alpha_{5}} .
$$

Then, $\left\{\alpha_{4}, \alpha_{5}\right\} \in V\left(s_{1}\right)$. Let $\alpha_{6}$ be an integer such that $\alpha_{6} \in$ $\mathcal{I}_{15} \backslash\left\{\alpha_{1}, \ldots, \alpha_{5}\right\}$ and

$$
\boldsymbol{s}_{2}=\boldsymbol{h}_{\alpha_{4}}+\boldsymbol{h}_{\alpha_{6}} .
$$

Then, $\left\{\alpha_{4}, \alpha_{6}\right\} \in V\left(s_{2}\right)$. From (70) and (71),

$$
\begin{aligned}
\boldsymbol{s}_{3} & =\boldsymbol{s}_{1}+\boldsymbol{s}_{2}=\left(\boldsymbol{h}_{\alpha_{4}}+\boldsymbol{h}_{\alpha_{5}}\right)+\left(\boldsymbol{h}_{\alpha_{4}}+\boldsymbol{h}_{\alpha_{6}}\right) \\
& =\boldsymbol{h}_{\alpha_{5}}+\boldsymbol{h}_{\alpha_{6}} .
\end{aligned}
$$

Hence, $\left\{\alpha_{5}, \alpha_{6}\right\} \in V\left(s_{3}\right)$. Let $\alpha_{7}$ be an integer such that $\alpha_{7} \in \mathcal{I}_{15} \backslash\left\{\alpha_{1}, \ldots, \alpha_{6}\right\}$ and

$$
\boldsymbol{s}_{1}=\boldsymbol{h}_{\alpha_{6}}+\boldsymbol{h}_{\alpha_{7}} .
$$

Then, $\left\{\alpha_{6}, \alpha_{7}\right\} \in V\left(s_{1}\right)$. From (70) and (73),

$$
\boldsymbol{h}_{\alpha_{4}}+\boldsymbol{h}_{\alpha_{5}}=\boldsymbol{h}_{\alpha_{6}}+\boldsymbol{h}_{\alpha_{7}} .
$$

From (74) and (71),

$$
\boldsymbol{h}_{\alpha_{5}}+\boldsymbol{h}_{\alpha_{7}}=\boldsymbol{h}_{\alpha_{4}}+\boldsymbol{h}_{\alpha_{6}}=\boldsymbol{s}_{2} .
$$

Therefore, $\left\{\alpha_{5}, \alpha_{7}\right\} \in V\left(\boldsymbol{s}_{2}\right)$. From (74) and (72),

$$
\boldsymbol{h}_{\alpha_{4}}+\boldsymbol{h}_{\alpha_{7}}=\boldsymbol{h}_{\alpha_{5}}+\boldsymbol{h}_{\alpha_{6}}=\boldsymbol{s}_{3} .
$$

Hence, $\left\{\alpha_{4}, \alpha_{7}\right\} \in V\left(s_{3}\right)$. Let $\alpha_{8}$ be an integer such that $\alpha_{8} \in \mathcal{I}_{15} \backslash\left\{\alpha_{1}, \ldots, \alpha_{7}\right\}$, and let $\alpha_{9}$ be an integer such that $\alpha_{9} \in \mathcal{I}_{15} \backslash\left\{\alpha_{1}, \ldots, \alpha_{8}\right\}$ and

$$
\boldsymbol{s}_{1}=\boldsymbol{h}_{\alpha_{8}}+\boldsymbol{h}_{\alpha_{9}} .
$$


Then, $\left\{\alpha_{8}, \alpha_{9}\right\} \in V\left(s_{1}\right)$. Let $\alpha_{10}$ be an integer such that $\alpha_{10} \in \mathcal{I}_{15} \backslash\left\{\alpha_{1}, \ldots, \alpha_{9}\right\}$ and

$$
\boldsymbol{s}_{2}=\boldsymbol{h}_{\alpha_{8}}+\boldsymbol{h}_{\alpha_{10}} \text {. }
$$

Then, $\left\{\alpha_{8}, \alpha_{10}\right\} \in V\left(s_{2}\right)$. From (77) and (78),

$$
\begin{aligned}
\boldsymbol{s}_{3} & =\boldsymbol{s}_{1}+\boldsymbol{s}_{2}=\left(\boldsymbol{h}_{\alpha_{8}}+\boldsymbol{h}_{\alpha_{9}}\right)+\left(\boldsymbol{h}_{\alpha_{8}}+\boldsymbol{h}_{\alpha_{10}}\right) \\
& =\boldsymbol{h}_{\alpha_{9}}+\boldsymbol{h}_{\alpha_{10}} .
\end{aligned}
$$

Therefore, $\left\{\alpha_{9}, \alpha_{10}\right\} \in V\left(s_{3}\right)$. Let $\alpha_{11}$ be an integer such that $\alpha_{11} \in \mathcal{I}_{15} \backslash\left\{\alpha_{1}, \ldots, \alpha_{10}\right\}$ and

$$
\boldsymbol{s}_{1}=\boldsymbol{h}_{\alpha_{10}}+\boldsymbol{h}_{\alpha_{11}} \text {. }
$$

Then, $\left\{\alpha_{10}, \alpha_{11}\right\} \in V\left(s_{1}\right)$. From (77) and (80),

$$
\boldsymbol{h}_{\alpha_{8}}+\boldsymbol{h}_{\alpha_{9}}=\boldsymbol{h}_{\alpha_{10}}+\boldsymbol{h}_{\alpha_{11}} \text {. }
$$

From (81) and (78),

$$
\boldsymbol{h}_{\alpha_{9}}+\boldsymbol{h}_{\alpha_{11}}=\boldsymbol{h}_{\alpha_{8}}+\boldsymbol{h}_{\alpha_{10}}=\boldsymbol{s}_{2} .
$$

Hence, $\left\{\alpha_{9}, \alpha_{11}\right\} \in V\left(s_{2}\right)$. From (81) and (79),

$$
\boldsymbol{h}_{\alpha_{8}}+\boldsymbol{h}_{\alpha_{11}}=\boldsymbol{h}_{\alpha_{9}}+\boldsymbol{h}_{\alpha_{10}}=\boldsymbol{s}_{3} .
$$

Therefore, $\left\{\alpha_{8}, \alpha_{11}\right\} \in V\left(s_{3}\right)$. Let $\alpha_{12}$ be an integer such that $\alpha_{12} \in \mathcal{I}_{15} \backslash\left\{\alpha_{1}, \ldots, \alpha_{11}\right\}$, and let $\alpha_{13}$ be an integer such that $\alpha_{13} \in \mathcal{I}_{15} \backslash\left\{\alpha_{1}, \ldots, \alpha_{12}\right\}$ and

$$
\boldsymbol{s}_{1}=\boldsymbol{h}_{\alpha_{12}}+\boldsymbol{h}_{\alpha_{13}} .
$$

Then, $\left\{\alpha_{12}, \alpha_{13}\right\} \in V\left(s_{1}\right)$. Let $\alpha_{14}$ be an integer such that $\alpha_{14} \in \mathcal{I}_{15} \backslash\left\{\alpha_{1}, \ldots, \alpha_{13}\right\}$ and

$$
\boldsymbol{s}_{2}=\boldsymbol{h}_{\alpha_{12}}+\boldsymbol{h}_{\alpha_{14}} .
$$

Then, $\left\{\alpha_{12}, \alpha_{14}\right\} \in V\left(s_{2}\right)$. From (84) and (85),

$$
\begin{aligned}
\boldsymbol{s}_{3} & =\boldsymbol{s}_{1}+\boldsymbol{s}_{2}=\left(\boldsymbol{h}_{\alpha_{12}}+\boldsymbol{h}_{\alpha_{13}}\right)+\left(\boldsymbol{h}_{\alpha_{12}}+\boldsymbol{h}_{\alpha_{14}}\right) \\
& =\boldsymbol{h}_{\alpha_{13}}+\boldsymbol{h}_{\alpha_{14}} .
\end{aligned}
$$

Hence, $\left\{\alpha_{13}, \alpha_{14}\right\} \in V\left(s_{3}\right)$. Let $\alpha_{15}$ be an integer such that $\alpha_{15} \in \mathcal{I}_{15} \backslash\left\{\alpha_{1}, \ldots, \alpha_{14}\right\}$ and

$$
\boldsymbol{s}_{1}=\boldsymbol{h}_{\alpha_{14}}+\boldsymbol{h}_{\alpha_{15}} \text {. }
$$

Then, $\left\{\alpha_{14}, \alpha_{15}\right\} \in V\left(s_{1}\right)$. From (84) and 87),

$$
\boldsymbol{h}_{\alpha_{12}}+\boldsymbol{h}_{\alpha_{13}}=\boldsymbol{h}_{\alpha_{14}}+\boldsymbol{h}_{\alpha_{15}} \text {. }
$$

From (88) and (85),

$$
\boldsymbol{h}_{\alpha_{13}}+\boldsymbol{h}_{\alpha_{15}}=\boldsymbol{h}_{\alpha_{12}}+\boldsymbol{h}_{\alpha_{14}}=\boldsymbol{s}_{2} .
$$

Therefore, $\left\{\alpha_{13}, \alpha_{15}\right\} \in V\left(s_{2}\right)$. From (88) and (86),

$$
\boldsymbol{h}_{\alpha_{12}}+\boldsymbol{h}_{\alpha_{15}}=\boldsymbol{h}_{\alpha_{13}}+\boldsymbol{h}_{\alpha_{14}}=\boldsymbol{s}_{3} \text {. }
$$

Hence, $\left\{\alpha_{12}, \alpha_{15}\right\} \in V\left(s_{3}\right)$. Let $\sigma$ be the permutation of $\mathcal{I}_{15}$ such that $\sigma(j)=\alpha_{j}$ for each $j \in \mathcal{I}_{15}$. Then, $\sigma$ satisfies (29), (30), and (31).

Theorem 6: Let $r=4$. For any $\boldsymbol{s}_{1}, \boldsymbol{s}_{2}, \boldsymbol{s}_{3}, \boldsymbol{s}_{4} \in\{0,1\}^{4} \backslash$ $\{0000\}\left(s_{i} \neq s_{i^{\prime}}\right.$ for any $\left.i, i^{\prime} \in \mathcal{I}_{4}, i \neq i^{\prime}\right)$, we have the permutation $\sigma$ of $\mathcal{I}_{15}$, which satisfies the following conditions: (a) If $s_{1}, s_{2}, s_{3}$, and $s_{4}$ are linearly independent,

$$
\begin{aligned}
V\left(s_{1}\right)= & \{\{\sigma(1)\},\{\sigma(2), \sigma(3)\},\{\sigma(4), \sigma(5)\}, \\
& \{\sigma(6), \sigma(7)\},\{\sigma(8), \sigma(9)\},\{\sigma(10), \sigma(11)\}, \\
V\left(s_{2}\right)= & \{\{\sigma(2)\},\{\sigma(1)\},\{\sigma(14), \sigma(15)\}\} \\
& \{\sigma(5), \sigma(7)\},\{\sigma(8), \sigma(10)\},\{\sigma(9), \sigma(11)\}, \\
& \{\sigma(12), \sigma(14)\},\{\sigma(13), \sigma(15)\}\}, \\
V\left(s_{3}\right)= & \{\{\sigma(4)\},\{\sigma(1), \sigma(5)\},\{\sigma(2), \sigma(6)\}, \\
& \{\sigma(3), \sigma(7)\},\{\sigma(8), \sigma(12)\},\{\sigma(9), \sigma(13)\}, \\
& \{\sigma(10), \sigma(14)\},\{\sigma(11), \sigma(15)\}\}, \\
V\left(s_{4}\right)= & \{\{\sigma(8)\},\{\sigma(1), \sigma(9)\},\{\sigma(2), \sigma(10)\}, \\
& \{\sigma(3), \sigma(11)\},\{\sigma(4), \sigma(12)\},\{\sigma(5), \sigma(13)\}, \\
& \{\sigma(6), \sigma(14)\},\{\sigma(7), \sigma(15)\}\}
\end{aligned}
$$

(b) If there exists the permutation $\tau$ of $\mathcal{I}_{4}$ such that $\boldsymbol{s}_{\tau(1)}+$ $\boldsymbol{s}_{\tau(2)}=\boldsymbol{s}_{\tau(3)}$,

$$
\begin{aligned}
V\left(\boldsymbol{s}_{\tau(1)}\right)= & \{\{\sigma(1)\},\{\sigma(2), \sigma(3)\},\{\sigma(4), \sigma(5)\}, \\
& \{\sigma(6), \sigma(7)\},\{\sigma(8), \sigma(9)\},\{\sigma(10), \sigma(11)\}, \\
& \{\sigma(12), \sigma(13)\},\{\sigma(14), \sigma(15)\}\}, \\
V\left(\boldsymbol{s}_{\tau(2)}\right)= & \{\{\sigma(2)\},\{\sigma(1), \sigma(3)\},\{\sigma(4), \sigma(6)\}, \\
& \{\sigma(5), \sigma(7)\},\{\sigma(8), \sigma(10)\},\{\sigma(9), \sigma(11)\}, \\
& \{\sigma(12), \sigma(14)\},\{\sigma(13), \sigma(15)\}\}, \\
V\left(\boldsymbol{s}_{\tau(3)}\right)= & \{\{\sigma(3)\},\{\sigma(1), \sigma(2)\},\{\sigma(4), \sigma(7)\}, \\
& \{\sigma(5), \sigma(6)\},\{\sigma(8), \sigma(11)\},\{\sigma(9), \sigma(10)\}, \\
& \{\sigma(12), \sigma(15)\},\{\sigma(13), \sigma(14)\}\}, \\
V\left(\boldsymbol{s}_{\tau(4)}\right)= & \{\{\sigma(4)\},\{\sigma(1), \sigma(5)\},\{\sigma(2), \sigma(6)\}, \\
& \{\sigma(3), \sigma(7)\},\{\sigma(8), \sigma(12)\},\{\sigma(9), \sigma(13)\}, \\
& \{\sigma(10), \sigma(14)\},\{\sigma(11), \sigma(15)\}\} .
\end{aligned}
$$

(c) If $s_{1}+s_{2}=s_{3}+s_{4}$,

$$
\begin{aligned}
V\left(s_{1}\right)= & \{\{\sigma(1)\},\{\sigma(2), \sigma(3)\},\{\sigma(4), \sigma(5)\} \\
& \{\sigma(6), \sigma(7)\},\{\sigma(8), \sigma(9)\},\{\sigma(10), \sigma(11)\} \\
& \{\sigma(12), \sigma(13)\},\{\sigma(14), \sigma(15)\}\} \\
V\left(s_{2}\right)= & \{\{\sigma(2)\},\{\sigma(1), \sigma(3)\},\{\sigma(4), \sigma(6)\} \\
& \{\sigma(5), \sigma(7)\},\{\sigma(8), \sigma(10)\},\{\sigma(9), \sigma(11)\} \\
& \{\sigma(12), \sigma(14)\},\{\sigma(13), \sigma(15)\}\} \\
V\left(s_{3}\right)= & \{\{\sigma(4)\},\{\sigma(1), \sigma(5)\},\{\sigma(2), \sigma(6)\} \\
& \{\sigma(3), \sigma(7)\},\{\sigma(8), \sigma(12)\},\{\sigma(9), \sigma(13)\}, \\
& \{\sigma(10), \sigma(14)\},\{\sigma(11), \sigma(15)\}\} \\
V\left(s_{4}\right)= & \{\{\sigma(7)\},\{\sigma(1), \sigma(6)\},\{\sigma(2), \sigma(5)\} \\
& \{\sigma(3), \sigma(4)\},\{\sigma(8), \sigma(15)\},\{\sigma(9), \sigma(14)\} \\
& \{\sigma(10), \sigma(13)\},\{\sigma(11), \sigma(12)\}\}
\end{aligned}
$$

Proof: Let $\alpha_{1}$ be an integer such that $\alpha_{1} \in \mathcal{I}_{15}$ and

$$
\boldsymbol{s}_{1}=\boldsymbol{h}_{\alpha_{1}} \text {. }
$$


Let $\alpha_{2}$ be an integer such that $\alpha_{2} \in \mathcal{I}_{15}$ and

$$
s_{2}=h_{\alpha_{2}} .
$$

Then, $\left\{\alpha_{1}\right\} \in V\left(\boldsymbol{s}_{1}\right),\left\{\alpha_{2}\right\} \in V\left(\boldsymbol{s}_{2}\right)$. Clearly, $\alpha_{1} \neq \alpha_{2}$. Let $\alpha_{3}$ be an integer such that $\alpha_{3} \in \mathcal{I}_{15} \backslash\left\{\alpha_{1}, \alpha_{2}\right\}$ and

$$
\boldsymbol{s}_{1}=\boldsymbol{h}_{\alpha_{2}}+\boldsymbol{h}_{\alpha_{3}} \text {. }
$$

Then, $\left\{\alpha_{2}, \alpha_{3}\right\} \in V\left(s_{1}\right)$. From (103), (105), and (104),

$$
\begin{aligned}
\boldsymbol{h}_{\alpha_{1}}+\boldsymbol{h}_{\alpha_{3}} & =\boldsymbol{s}_{1}+\boldsymbol{h}_{\alpha_{3}}=\left(\boldsymbol{h}_{\alpha_{2}}+\boldsymbol{h}_{\alpha_{3}}\right)+\boldsymbol{h}_{\alpha_{3}} \\
& =\boldsymbol{h}_{\alpha_{2}}=\boldsymbol{s}_{2} .
\end{aligned}
$$

Hence, $\left\{\alpha_{1}, \alpha_{3}\right\} \in V\left(s_{2}\right)$.

(a) Let $\alpha_{4}$ be an integer such that $\alpha_{4} \in \mathcal{I}_{15}$ and

$$
s_{3}=h_{\alpha_{4}} .
$$

Then, $\left\{\alpha_{4}\right\} \in V\left(s_{3}\right)$. Clearly, $\alpha_{4} \notin\left\{\alpha_{1}, \alpha_{2}\right\}$. From (107), (105), and (104),

$$
\boldsymbol{h}_{\alpha_{4}}=\boldsymbol{s}_{3} \neq \boldsymbol{s}_{1}+\boldsymbol{s}_{2}=\left(\boldsymbol{h}_{\alpha_{2}}+\boldsymbol{h}_{\alpha_{3}}\right)+\boldsymbol{h}_{\alpha_{2}}=\boldsymbol{h}_{\alpha_{3}} \text {. }
$$

Therefore, $\alpha_{4} \neq \alpha_{3}$. Let $\alpha_{5}$ be an integer such that $\alpha_{5} \in$ $\mathcal{I}_{15} \backslash\left\{\alpha_{1}, \ldots, \alpha_{4}\right\}$ and

$$
\boldsymbol{s}_{1}=\boldsymbol{h}_{\alpha_{4}}+\boldsymbol{h}_{\alpha_{5}} .
$$

Then, $\left\{\alpha_{4}, \alpha_{5}\right\} \in V\left(s_{1}\right)$. From (103) and (109),

$$
\boldsymbol{h}_{\alpha_{1}}=\boldsymbol{h}_{\alpha_{4}}+\boldsymbol{h}_{\alpha_{5}} .
$$

From (110) and (107),

$$
\boldsymbol{h}_{\alpha_{1}}+\boldsymbol{h}_{\alpha_{5}}=\boldsymbol{h}_{\alpha_{4}}=\boldsymbol{s}_{3} .
$$

Hence, $\left\{\alpha_{1}, \alpha_{5}\right\} \in V\left(s_{3}\right)$. Let $\alpha_{6}$ be an integer such that $\alpha_{6} \in \mathcal{I}_{15} \backslash\left\{\alpha_{1}, \ldots, \alpha_{5}\right\}$ and

$$
\boldsymbol{s}_{2}=\boldsymbol{h}_{\alpha_{4}}+\boldsymbol{h}_{\alpha_{6}} \text {. }
$$

Then, $\left\{\alpha_{4}, \alpha_{6}\right\} \in V\left(s_{2}\right)$. From (104) and (112),

$$
\boldsymbol{h}_{\alpha_{2}}=\boldsymbol{h}_{\alpha_{4}}+\boldsymbol{h}_{\alpha_{6}} \text {. }
$$

From (113) and (107),

$$
\boldsymbol{h}_{\alpha_{2}}+\boldsymbol{h}_{\alpha_{6}}=\boldsymbol{h}_{\alpha_{4}}=\boldsymbol{s}_{3} .
$$

Therefore, $\left\{\alpha_{2}, \alpha_{6}\right\} \in V\left(s_{3}\right)$. Let $\alpha_{7}$ be an integer such that $\alpha_{7} \in \mathcal{I}_{15} \backslash\left\{\alpha_{1}, \ldots, \alpha_{6}\right\}$ and

$$
\boldsymbol{s}_{1}=\boldsymbol{h}_{\alpha_{6}}+\boldsymbol{h}_{\alpha_{7}} .
$$

Then, $\left\{\alpha_{6}, \alpha_{7}\right\} \in V\left(s_{1}\right)$. From (109) and (115),

$$
\boldsymbol{h}_{\alpha_{4}}+\boldsymbol{h}_{\alpha_{5}}=\boldsymbol{h}_{\alpha_{6}}+\boldsymbol{h}_{\alpha_{7}} \text {. }
$$

From (116) and (112),

$$
\boldsymbol{h}_{\alpha_{5}}+\boldsymbol{h}_{\alpha_{7}}=\boldsymbol{h}_{\alpha_{4}}+\boldsymbol{h}_{\alpha_{6}}=\boldsymbol{s}_{2} .
$$

Hence, $\left\{\alpha_{5}, \alpha_{7}\right\} \in V\left(s_{2}\right)$. From (105) and (115),

$$
\boldsymbol{h}_{\alpha_{2}}+\boldsymbol{h}_{\alpha_{3}}=\boldsymbol{h}_{\alpha_{6}}+\boldsymbol{h}_{\alpha_{7}} \text {. }
$$

From (118) and (114),

$$
\boldsymbol{h}_{\alpha_{3}}+\boldsymbol{h}_{\alpha_{7}}=\boldsymbol{h}_{\alpha_{2}}+\boldsymbol{h}_{\alpha_{6}}=s_{3} .
$$

Therefore, $\left\{\alpha_{3}, \alpha_{7}\right\} \in V\left(s_{3}\right)$. Let $\alpha_{8}$ be an integer such that $\alpha_{8} \in \mathcal{I}_{15}$ and

$$
s_{4}=h_{\alpha_{8}} .
$$

Then, $\left\{\alpha_{8}\right\} \in V\left(s_{4}\right)$. Clearly, $\alpha_{8} \notin\left\{\alpha_{1}, \ldots, \alpha_{4}\right\}$. From (120), (109), and (107),

$$
\boldsymbol{h}_{\alpha_{8}}=\boldsymbol{s}_{4} \neq \boldsymbol{s}_{1}+\boldsymbol{s}_{3}=\left(\boldsymbol{h}_{\alpha_{4}}+\boldsymbol{h}_{\alpha_{5}}\right)+\boldsymbol{h}_{\alpha_{4}}=\boldsymbol{h}_{\alpha_{5}} .
$$

From (120), 1112), and (107),

$$
\boldsymbol{h}_{\alpha_{8}}=\boldsymbol{s}_{4} \neq \boldsymbol{s}_{2}+\boldsymbol{s}_{3}=\left(\boldsymbol{h}_{\alpha_{4}}+\boldsymbol{h}_{\alpha_{6}}\right)+\boldsymbol{h}_{\alpha_{4}}=\boldsymbol{h}_{\alpha_{6}} \text {. }
$$

From (120), (115), (112), and (107),

$$
\begin{aligned}
& \boldsymbol{h}_{\alpha_{8}}=\boldsymbol{s}_{4} \\
\neq \quad & \boldsymbol{s}_{1}+\boldsymbol{s}_{2}+\boldsymbol{s}_{3}=\left(\boldsymbol{h}_{\alpha_{6}}+\boldsymbol{h}_{\alpha_{7}}\right)+\left(\boldsymbol{h}_{\alpha_{4}}+\boldsymbol{h}_{\alpha_{6}}\right)+\boldsymbol{h}_{\alpha_{4}} \\
= & \boldsymbol{h}_{\alpha_{7}} .
\end{aligned}
$$

From (121), (122), and (123), $\alpha_{8} \notin\left\{\alpha_{1}, \ldots, \alpha_{7}\right\}$. Let $\alpha_{9}$ be an integer such that $\alpha_{9} \in \mathcal{I}_{15} \backslash\left\{\alpha_{1}, \ldots, \alpha_{8}\right\}$ and

$$
\boldsymbol{s}_{1}=\boldsymbol{h}_{\alpha_{8}}+\boldsymbol{h}_{\alpha_{9}} .
$$

Then, $\left\{\alpha_{8}, \alpha_{9}\right\} \in V\left(s_{1}\right)$. From (103) and (124),

$$
\boldsymbol{h}_{\alpha_{1}}=\boldsymbol{h}_{\alpha_{8}}+\boldsymbol{h}_{\alpha_{9}} \text {. }
$$

From (125) and (120),

$$
\boldsymbol{h}_{\alpha_{1}}+\boldsymbol{h}_{\alpha_{9}}=\boldsymbol{h}_{\alpha_{8}}=\boldsymbol{s}_{4} \text {. }
$$

Hence, $\left\{\alpha_{1}, \alpha_{9}\right\} \in V\left(s_{4}\right)$. Let $\alpha_{10}$ be an integer such that $\alpha_{10} \in \mathcal{I}_{15} \backslash\left\{\alpha_{1}, \ldots, \alpha_{9}\right\}$ and

$$
\boldsymbol{s}_{2}=\boldsymbol{h}_{\alpha_{8}}+\boldsymbol{h}_{\alpha_{10}} .
$$

Then, $\left\{\alpha_{8}, \alpha_{10}\right\} \in V\left(s_{2}\right)$. From (104) and (127),

$$
\boldsymbol{h}_{\alpha_{2}}=\boldsymbol{h}_{\alpha_{8}}+\boldsymbol{h}_{\alpha_{10}} .
$$

From (128) and (120),

$$
\boldsymbol{h}_{\alpha_{2}}+\boldsymbol{h}_{\alpha_{10}}=\boldsymbol{h}_{\alpha_{8}}=\boldsymbol{s}_{4} .
$$

Therefore, $\left\{\alpha_{2}, \alpha_{10}\right\} \in V\left(s_{4}\right)$. Let $\alpha_{11}$ be an integer such that $\alpha_{11} \in \mathcal{I}_{15} \backslash\left\{\alpha_{1}, \ldots, \alpha_{10}\right\}$ and

$$
\boldsymbol{s}_{1}=\boldsymbol{h}_{\alpha_{10}}+\boldsymbol{h}_{\alpha_{11}} \text {. }
$$

Then, $\left\{\alpha_{10}, \alpha_{11}\right\} \in V\left(\boldsymbol{s}_{1}\right)$. From (124) and (130),

$$
\boldsymbol{h}_{\alpha_{8}}+\boldsymbol{h}_{\alpha_{9}}=\boldsymbol{h}_{\alpha_{10}}+\boldsymbol{h}_{\alpha_{11}} .
$$

From (131) and (127),

$$
\boldsymbol{h}_{\alpha_{9}}+\boldsymbol{h}_{\alpha_{11}}=\boldsymbol{h}_{\alpha_{8}}+\boldsymbol{h}_{\alpha_{10}}=\boldsymbol{s}_{2} .
$$

Hence, $\left\{\alpha_{9}, \alpha_{11}\right\} \in V\left(s_{2}\right)$. From (106) and (132),

$$
\boldsymbol{h}_{\alpha_{1}}+\boldsymbol{h}_{\alpha_{3}}=\boldsymbol{h}_{\alpha_{9}}+\boldsymbol{h}_{\alpha_{11}} .
$$

From (133) and (126),

$$
\boldsymbol{h}_{\alpha_{3}}+\boldsymbol{h}_{\alpha_{11}}=\boldsymbol{h}_{\alpha_{1}}+\boldsymbol{h}_{\alpha_{9}}=\boldsymbol{s}_{4} .
$$


Therefore, $\left\{\alpha_{3}, \alpha_{11}\right\} \in V\left(s_{4}\right)$. Let $\alpha_{12}$ be an integer such that $\alpha_{12} \in \mathcal{I}_{15} \backslash\left\{\alpha_{1}, \ldots, \alpha_{10}\right\}$ and

$$
\boldsymbol{s}_{3}=\boldsymbol{h}_{\alpha_{8}}+\boldsymbol{h}_{\alpha_{12}} \text {. }
$$

Then, $\left\{\alpha_{8}, \alpha_{12}\right\} \in V\left(s_{3}\right)$. From (135), (124), and (132),

$$
\begin{aligned}
& \boldsymbol{h}_{\alpha_{8}}+\boldsymbol{h}_{\alpha_{12}}=\boldsymbol{s}_{3} \\
\neq \quad & \boldsymbol{s}_{1}+\boldsymbol{s}_{2}=\left(\boldsymbol{h}_{\alpha_{8}}+\boldsymbol{h}_{\alpha_{9}}\right)+\left(\boldsymbol{h}_{\alpha_{9}}+\boldsymbol{h}_{\alpha_{11}}\right) \\
& =\boldsymbol{h}_{\alpha_{8}}+\boldsymbol{h}_{\alpha_{11}} .
\end{aligned}
$$

Therefore, $\alpha_{12} \neq \alpha_{11}$. From (107) and (135),

$$
\boldsymbol{h}_{\alpha_{4}}=\boldsymbol{h}_{\alpha_{8}}+\boldsymbol{h}_{\alpha_{12}} \text {. }
$$

From (137) and (120),

$$
\boldsymbol{h}_{\alpha_{4}}+\boldsymbol{h}_{\alpha_{12}}=\boldsymbol{h}_{\alpha_{8}}=\boldsymbol{s}_{4} \text {. }
$$

Hence, $\left\{\alpha_{4}, \alpha_{12}\right\} \in V\left(\boldsymbol{s}_{4}\right)$. Let $\alpha_{13}$ be an integer such that $\alpha_{13} \in \mathcal{I}_{15} \backslash\left\{\alpha_{1}, \ldots, \alpha_{12}\right\}$ and

$$
\boldsymbol{s}_{1}=\boldsymbol{h}_{\alpha_{12}}+\boldsymbol{h}_{\alpha_{13}} .
$$

Then, $\left\{\alpha_{12}, \alpha_{13}\right\} \in V\left(s_{1}\right)$. From (124) and (139),

$$
\boldsymbol{h}_{\alpha_{8}}+\boldsymbol{h}_{\alpha_{9}}=\boldsymbol{h}_{\alpha_{12}}+\boldsymbol{h}_{\alpha_{13}} \text {. }
$$

From (140) and (135),

$$
\boldsymbol{h}_{\alpha_{9}}+\boldsymbol{h}_{\alpha_{13}}=\boldsymbol{h}_{\alpha_{8}}+\boldsymbol{h}_{\alpha_{12}}=\boldsymbol{s}_{3} .
$$

Therefore, $\left\{\alpha_{9}, \alpha_{13}\right\} \in V\left(s_{3}\right)$. From (109) and (139),

$$
\boldsymbol{h}_{\alpha_{4}}+\boldsymbol{h}_{\alpha_{5}}=\boldsymbol{h}_{\alpha_{12}}+\boldsymbol{h}_{\alpha_{13}} \text {. }
$$

From 142 and (138),

$$
\boldsymbol{h}_{\alpha_{5}}+\boldsymbol{h}_{\alpha_{13}}=\boldsymbol{h}_{\alpha_{4}}+\boldsymbol{h}_{\alpha_{12}}=\boldsymbol{s}_{4} .
$$

Hence, $\left\{\alpha_{5}, \alpha_{13}\right\} \in V\left(s_{4}\right)$. Let $\alpha_{14}$ be an integer such that $\alpha_{14} \in \mathcal{I}_{15} \backslash\left\{\alpha_{1}, \ldots, \alpha_{13}\right\}$ and

$$
\boldsymbol{s}_{2}=\boldsymbol{h}_{\alpha_{12}}+\boldsymbol{h}_{\alpha_{14}} \text {. }
$$

Then, $\left\{\alpha_{12}, \alpha_{14}\right\} \in V\left(s_{2}\right)$. From (127) and (144),

$$
\boldsymbol{h}_{\alpha_{8}}+\boldsymbol{h}_{\alpha_{10}}=\boldsymbol{h}_{\alpha_{12}}+\boldsymbol{h}_{\alpha_{14}} .
$$

From (145) and (135),

$$
\boldsymbol{h}_{\alpha_{10}}+\boldsymbol{h}_{\alpha_{14}}=\boldsymbol{h}_{\alpha_{8}}+\boldsymbol{h}_{\alpha_{12}}=\boldsymbol{s}_{3} .
$$

Therefore, $\left\{\alpha_{10}, \alpha_{14}\right\} \in V\left(s_{3}\right)$. From (112) and (144),

$$
\boldsymbol{h}_{\alpha_{4}}+\boldsymbol{h}_{\alpha_{6}}=\boldsymbol{h}_{\alpha_{12}}+\boldsymbol{h}_{\alpha_{14}} \text {. }
$$

From (147) and (138),

$$
\boldsymbol{h}_{\alpha_{6}}+\boldsymbol{h}_{\alpha_{14}}=\boldsymbol{h}_{\alpha_{4}}+\boldsymbol{h}_{\alpha_{12}}=\boldsymbol{s}_{4} .
$$

Hence, $\left\{\alpha_{6}, \alpha_{14}\right\} \in V\left(s_{4}\right)$. Let $\alpha_{15}$ be an integer such that $\alpha_{15} \in \mathcal{I}_{15} \backslash\left\{\alpha_{1}, \ldots, \alpha_{14}\right\}$ and

$$
\boldsymbol{s}_{1}=\boldsymbol{h}_{\alpha_{14}}+\boldsymbol{h}_{\alpha_{15}} \text {. }
$$

Then, $\left\{\alpha_{14}, \alpha_{15}\right\} \in V\left(s_{1}\right)$. From (139) and (149),

$$
\boldsymbol{h}_{\alpha_{12}}+\boldsymbol{h}_{\alpha_{13}}=\boldsymbol{h}_{\alpha_{14}}+\boldsymbol{h}_{\alpha_{15}} .
$$

From (150) and (144),

$$
\boldsymbol{h}_{\alpha_{13}}+\boldsymbol{h}_{\alpha_{15}}=\boldsymbol{h}_{\alpha_{12}}+\boldsymbol{h}_{\alpha_{14}}=\boldsymbol{s}_{2} .
$$

Therefore, $\left\{\alpha_{13}, \alpha_{15}\right\} \in V\left(s_{2}\right)$. From (130) and (149),

$$
\boldsymbol{h}_{\alpha_{10}}+\boldsymbol{h}_{\alpha_{11}}=\boldsymbol{h}_{\alpha_{14}}+\boldsymbol{h}_{\alpha_{15}} .
$$

From (152) and (146),

$$
\boldsymbol{h}_{\alpha_{11}}+\boldsymbol{h}_{\alpha_{15}}=\boldsymbol{h}_{\alpha_{10}}+\boldsymbol{h}_{\alpha_{14}}=\boldsymbol{s}_{3} .
$$

Hence, $\left\{\alpha_{11}, \alpha_{15}\right\} \in V\left(s_{3}\right)$. From (115) and (149),

$$
\boldsymbol{h}_{\alpha_{6}}+\boldsymbol{h}_{\alpha_{7}}=\boldsymbol{h}_{\alpha_{14}}+\boldsymbol{h}_{\alpha_{15}} .
$$

From (154) and (148),

$$
\boldsymbol{h}_{\alpha_{7}}+\boldsymbol{h}_{\alpha_{15}}=\boldsymbol{h}_{\alpha_{6}}+\boldsymbol{h}_{\alpha_{14}}=\boldsymbol{s}_{4} .
$$

Therefore, $\left\{\alpha_{7}, \alpha_{15}\right\} \in V\left(\boldsymbol{s}_{4}\right)$. Let $\sigma$ be the permutation of $\mathcal{I}_{15}$ such that $\sigma(j)=\alpha_{j}$ for each $j \in \mathcal{I}_{15}$. Then, $\sigma$ satisfies (91), 92), 93), and 94).

(b) For simplicity, suppose $s_{1}+s_{2}=s_{3}$. From (105) and (104),

$$
\boldsymbol{s}_{3}=\boldsymbol{s}_{1}+\boldsymbol{s}_{2}=\left(\boldsymbol{h}_{\alpha_{2}}+\boldsymbol{h}_{\alpha_{3}}\right)+\boldsymbol{h}_{\alpha_{2}}=\boldsymbol{h}_{\alpha_{3}} .
$$

Hence, $\left\{\alpha_{3}\right\} \in V\left(s_{3}\right)$. From (103) and (104),

$$
\boldsymbol{h}_{\alpha_{1}}+\boldsymbol{h}_{\alpha_{2}}=s_{1}+s_{2}=s_{3} .
$$

Therefore, $\left\{\alpha_{1}, \alpha_{2}\right\} \in V\left(s_{3}\right)$. Let $\alpha_{4}$ be an integer such that $\alpha_{4} \in \mathcal{I}_{15}$ and

$$
\boldsymbol{s}_{4}=\boldsymbol{h}_{\alpha_{4}} .
$$

Then, $\left\{\alpha_{4}\right\} \in V\left(\boldsymbol{s}_{4}\right)$. Clearly, $\alpha_{4} \notin\left\{\alpha_{1}, \alpha_{2}, \alpha_{3}\right\}$. Let $\alpha_{5}$ be an integer such that $\alpha_{5} \in \mathcal{I}_{15} \backslash\left\{\alpha_{1}, \ldots, \alpha_{4}\right\}$ and

$$
\boldsymbol{s}_{1}=\boldsymbol{h}_{\alpha_{4}}+\boldsymbol{h}_{\alpha_{5}} .
$$

Then, $\left\{\alpha_{4}, \alpha_{5}\right\} \in V\left(s_{1}\right)$. From (103) and (159),

$$
\boldsymbol{h}_{\alpha_{1}}=\boldsymbol{h}_{\alpha_{4}}+\boldsymbol{h}_{\alpha_{5}} .
$$

From (160) and (158),

$$
\boldsymbol{h}_{\alpha_{1}}+\boldsymbol{h}_{\alpha_{5}}=\boldsymbol{h}_{\alpha_{4}}=\boldsymbol{s}_{4} .
$$

Hence, $\left\{\alpha_{1}, \alpha_{5}\right\} \in V\left(s_{4}\right)$. Let $\alpha_{6}$ be an integer such that $\alpha_{6} \in \mathcal{I}_{15} \backslash\left\{\alpha_{1}, \ldots, \alpha_{5}\right\}$ and

$$
\boldsymbol{s}_{2}=\boldsymbol{h}_{\alpha_{4}}+\boldsymbol{h}_{\alpha_{6}} .
$$

Then, $\left\{\alpha_{4}, \alpha_{6}\right\} \in V\left(s_{2}\right)$. From (159) and (162),

$$
\begin{aligned}
\boldsymbol{s}_{3} & =\boldsymbol{s}_{1}+\boldsymbol{s}_{2}=\left(\boldsymbol{h}_{\alpha_{4}}+\boldsymbol{h}_{\alpha_{5}}\right)+\left(\boldsymbol{h}_{\alpha_{4}}+\boldsymbol{h}_{\alpha_{6}}\right) \\
& =\boldsymbol{h}_{\alpha_{5}}+\boldsymbol{h}_{\alpha_{6}} .
\end{aligned}
$$

Therefore, $\left\{\alpha_{5}, \alpha_{6}\right\} \in V\left(s_{3}\right)$. From (104) and (162),

$$
\boldsymbol{h}_{\alpha_{2}}=\boldsymbol{h}_{\alpha_{4}}+\boldsymbol{h}_{\alpha_{6}} .
$$

From (164) and (158),

$$
\boldsymbol{h}_{\alpha_{2}}+\boldsymbol{h}_{\alpha_{6}}=\boldsymbol{h}_{\alpha_{4}}=\boldsymbol{s}_{4} .
$$


Hence, $\left\{\alpha_{2}, \alpha_{6}\right\} \in V\left(s_{4}\right)$. Let $\alpha_{7}$ be an integer such that $\alpha_{7} \in \mathcal{I}_{15} \backslash\left\{\alpha_{1}, \ldots, \alpha_{6}\right\}$ and

$$
\boldsymbol{s}_{1}=\boldsymbol{h}_{\alpha_{6}}+\boldsymbol{h}_{\alpha_{7}} \text {. }
$$

Then, $\left\{\alpha_{6}, \alpha_{7}\right\} \in V\left(s_{1}\right)$. From (159) and (166),

$$
\boldsymbol{h}_{\alpha_{4}}+\boldsymbol{h}_{\alpha_{5}}=\boldsymbol{h}_{\alpha_{6}}+\boldsymbol{h}_{\alpha_{7}} \text {. }
$$

From (167) and (162),

$$
\boldsymbol{h}_{\alpha_{5}}+\boldsymbol{h}_{\alpha_{7}}=\boldsymbol{h}_{\alpha_{4}}+\boldsymbol{h}_{\alpha_{6}}=\boldsymbol{s}_{2} .
$$

Therefore, $\left\{\alpha_{5}, \alpha_{7}\right\} \in V\left(s_{2}\right)$. From (167) and (163),

$$
\boldsymbol{h}_{\alpha_{4}}+\boldsymbol{h}_{\alpha_{7}}=\boldsymbol{h}_{\alpha_{5}}+\boldsymbol{h}_{\alpha_{6}}=\boldsymbol{s}_{3} \text {. }
$$

Hence, $\left\{\alpha_{4}, \alpha_{7}\right\} \in V\left(s_{3}\right)$. From (105) and (166),

$$
\boldsymbol{h}_{\alpha_{2}}+\boldsymbol{h}_{\alpha_{3}}=\boldsymbol{h}_{\alpha_{6}}+\boldsymbol{h}_{\alpha_{7}} .
$$

From (170) and (165),

$$
\boldsymbol{h}_{\alpha_{3}}+\boldsymbol{h}_{\alpha_{7}}=\boldsymbol{h}_{\alpha_{2}}+\boldsymbol{h}_{\alpha_{6}}=\boldsymbol{s}_{4} .
$$

Therefore, $\left\{\alpha_{3}, \alpha_{7}\right\} \in V\left(\boldsymbol{s}_{4}\right)$. Let $\alpha_{8}$ be an integer such that $\alpha_{8} \in \mathcal{I}_{15} \backslash\left\{\alpha_{1}, \ldots, \alpha_{7}\right\}$, and let $\alpha_{9}$ be an integer such that $\alpha_{9} \in \mathcal{I}_{15} \backslash\left\{\alpha_{1}, \ldots, \alpha_{8}\right\}$ and

$$
\boldsymbol{s}_{1}=\boldsymbol{h}_{\alpha_{8}}+\boldsymbol{h}_{\alpha_{9}} .
$$

Then, $\left\{\alpha_{8}, \alpha_{9}\right\} \in V\left(s_{1}\right)$. Let $\alpha_{10}$ be an integer such that $\alpha_{10} \in \mathcal{I}_{15} \backslash\left\{\alpha_{1}, \ldots, \alpha_{9}\right\}$ and

$$
\boldsymbol{s}_{2}=\boldsymbol{h}_{\alpha_{8}}+\boldsymbol{h}_{\alpha_{10}} .
$$

Then, $\left\{\alpha_{8}, \alpha_{10}\right\} \in V\left(s_{2}\right)$. From (172) and (173),

$$
\begin{aligned}
\boldsymbol{s}_{3} & =\boldsymbol{s}_{1}+\boldsymbol{s}_{2}=\left(\boldsymbol{h}_{\alpha_{8}}+\boldsymbol{h}_{\alpha_{9}}\right)+\left(\boldsymbol{h}_{\alpha_{8}}+\boldsymbol{h}_{\alpha_{10}}\right) \\
& =\boldsymbol{h}_{\alpha_{9}}+\boldsymbol{h}_{\alpha_{10}} .
\end{aligned}
$$

Hence, $\left\{\alpha_{9}, \alpha_{10}\right\} \in V\left(s_{3}\right)$. Let $\alpha_{11}$ be an integer such that $\alpha_{11} \in \mathcal{I}_{15} \backslash\left\{\alpha_{1}, \ldots, \alpha_{10}\right\}$ and

$$
\boldsymbol{s}_{1}=\boldsymbol{h}_{\alpha_{10}}+\boldsymbol{h}_{\alpha_{11}} .
$$

Then, $\left\{\alpha_{10}, \alpha_{11}\right\} \in V\left(s_{1}\right)$. From (172) and (175),

$$
\boldsymbol{h}_{\alpha_{8}}+\boldsymbol{h}_{\alpha_{9}}=\boldsymbol{h}_{\alpha_{10}}+\boldsymbol{h}_{\alpha_{11}} .
$$

From (176) and (173),

$$
\boldsymbol{h}_{\alpha_{9}}+\boldsymbol{h}_{\alpha_{11}}=\boldsymbol{h}_{\alpha_{8}}+\boldsymbol{h}_{\alpha_{10}}=\boldsymbol{s}_{2} .
$$

Therefore, $\left\{\alpha_{9}, \alpha_{11}\right\} \in V\left(s_{2}\right)$. From (176) and (174),

$$
\boldsymbol{h}_{\alpha_{8}}+\boldsymbol{h}_{\alpha_{11}}=\boldsymbol{h}_{\alpha_{9}}+\boldsymbol{h}_{\alpha_{10}}=\boldsymbol{s}_{3} .
$$

Hence, $\left\{\alpha_{8}, \alpha_{11}\right\} \in V\left(s_{3}\right)$. Let $\alpha_{12}$ be an integer such that $\alpha_{12} \in \mathcal{I}_{15} \backslash\left\{\alpha_{1}, \ldots, \alpha_{11}\right\}$ and

$$
\boldsymbol{s}_{4}=\boldsymbol{h}_{\alpha_{8}}+\boldsymbol{h}_{\alpha_{12}} .
$$

Then, $\left\{\alpha_{8}, \alpha_{12}\right\} \in V\left(\boldsymbol{s}_{4}\right)$. Let $\alpha_{13}$ be an integer such that $\alpha_{13} \in \mathcal{I}_{15} \backslash\left\{\alpha_{1}, \ldots, \alpha_{12}\right\}$ and

$$
\boldsymbol{s}_{1}=\boldsymbol{h}_{\alpha_{12}}+\boldsymbol{h}_{\alpha_{13}} \text {. }
$$

Then, $\left\{\alpha_{12}, \alpha_{13}\right\} \in V\left(s_{1}\right)$. From (172) and (180),

$$
\boldsymbol{h}_{\alpha_{8}}+\boldsymbol{h}_{\alpha_{9}}=\boldsymbol{h}_{\alpha_{12}}+\boldsymbol{h}_{\alpha_{13}} .
$$

From (181) and (179),

$$
\boldsymbol{h}_{\alpha_{9}}+\boldsymbol{h}_{\alpha_{13}}=\boldsymbol{h}_{\alpha_{8}}+\boldsymbol{h}_{\alpha_{12}}=\boldsymbol{s}_{4} .
$$

Therefore, $\left\{\alpha_{9}, \alpha_{13}\right\} \in V\left(s_{4}\right)$. Let $\alpha_{14}$ be an integer such that $\alpha_{14} \in \mathcal{I}_{15} \backslash\left\{\alpha_{1}, \ldots, \alpha_{13}\right\}$ and

$$
\boldsymbol{s}_{2}=\boldsymbol{h}_{\alpha_{12}}+\boldsymbol{h}_{\alpha_{14}} .
$$

Then, $\left\{\alpha_{12}, \alpha_{14}\right\} \in V\left(s_{2}\right)$. From (180) and (183),

$$
\begin{aligned}
\boldsymbol{s}_{3} & =\boldsymbol{s}_{1}+\boldsymbol{s}_{2}=\left(\boldsymbol{h}_{\alpha_{12}}+\boldsymbol{h}_{\alpha_{13}}\right)+\left(\boldsymbol{h}_{\alpha_{12}}+\boldsymbol{h}_{\alpha_{14}}\right) \\
& =\boldsymbol{h}_{\alpha_{13}}+\boldsymbol{h}_{\alpha_{14}} .
\end{aligned}
$$

Hence, $\left\{\alpha_{13}, \alpha_{14}\right\} \in V\left(s_{3}\right)$. From (173) and (183),

$$
\boldsymbol{h}_{\alpha_{8}}+\boldsymbol{h}_{\alpha_{10}}=\boldsymbol{h}_{\alpha_{12}}+\boldsymbol{h}_{\alpha_{14}} .
$$

From (185) and (179),

$$
\boldsymbol{h}_{\alpha_{10}}+\boldsymbol{h}_{\alpha_{14}}=\boldsymbol{h}_{\alpha_{8}}+\boldsymbol{h}_{\alpha_{12}}=\boldsymbol{s}_{4} .
$$

Therefore, $\left\{\alpha_{10}, \alpha_{14}\right\} \in V\left(s_{4}\right)$. Let $\alpha_{15}$ be an integer such that $\alpha_{15} \in \mathcal{I}_{15} \backslash\left\{\alpha_{1}, \ldots, \alpha_{14}\right\}$ and

$$
\boldsymbol{s}_{1}=\boldsymbol{h}_{\alpha_{14}}+\boldsymbol{h}_{\alpha_{15}} .
$$

Then, $\left\{\alpha_{14}, \alpha_{15}\right\} \in V\left(\boldsymbol{s}_{1}\right)$. From (180) and 187),

$$
\boldsymbol{h}_{\alpha_{12}}+\boldsymbol{h}_{\alpha_{13}}=\boldsymbol{h}_{\alpha_{14}}+\boldsymbol{h}_{\alpha_{15}} .
$$

From (188) and (183),

$$
\boldsymbol{h}_{\alpha_{13}}+\boldsymbol{h}_{\alpha_{15}}=\boldsymbol{h}_{\alpha_{12}}+\boldsymbol{h}_{\alpha_{14}}=\boldsymbol{s}_{2} .
$$

Hence, $\left\{\alpha_{13}, \alpha_{15}\right\} \in V\left(s_{2}\right)$. From (188) and (184),

$$
\boldsymbol{h}_{\alpha_{12}}+\boldsymbol{h}_{\alpha_{15}}=\boldsymbol{h}_{\alpha_{13}}+\boldsymbol{h}_{\alpha_{14}}=\boldsymbol{s}_{3} \text {. }
$$

Therefore, $\left\{\alpha_{12}, \alpha_{15}\right\} \in V\left(s_{3}\right)$. From (175) and (187),

$$
\boldsymbol{h}_{\alpha_{10}}+\boldsymbol{h}_{\alpha_{11}}=\boldsymbol{h}_{\alpha_{14}}+\boldsymbol{h}_{\alpha_{15}} .
$$

From (191) and (186),

$$
\boldsymbol{h}_{\alpha_{11}}+\boldsymbol{h}_{\alpha_{15}}=\boldsymbol{h}_{\alpha_{10}}+\boldsymbol{h}_{\alpha_{14}}=\boldsymbol{s}_{4} .
$$

Hence, $\left\{\alpha_{11}, \alpha_{15}\right\} \in V\left(s_{4}\right)$. Let $\sigma$ be the permutation of $\mathcal{I}_{15}$ such that $\sigma(j)=\alpha_{j}$ for each $j \in \mathcal{I}_{15}$. Then, $\sigma$ satisfies (95), (96), 977), and (98).

(c) Let $\alpha_{4}$ be an integer such that $\alpha_{4} \in \mathcal{I}_{15}$ and

$$
\boldsymbol{s}_{3}=\boldsymbol{h}_{\alpha_{4}} .
$$

Then, $\left\{\alpha_{4}\right\} \in V\left(s_{3}\right)$. Clearly, $\alpha_{4} \notin\left\{\alpha_{1}, \alpha_{2}\right\}$. From (193), (105), and 104,

$$
\boldsymbol{h}_{\alpha_{4}}=\boldsymbol{s}_{3} \neq \boldsymbol{s}_{1}+\boldsymbol{s}_{2}=\left(\boldsymbol{h}_{\alpha_{2}}+\boldsymbol{h}_{\alpha_{3}}\right)+\boldsymbol{h}_{\alpha_{2}}=\boldsymbol{h}_{\alpha_{3}}
$$

Therefore, $\alpha_{4} \neq \alpha_{3}$. From (103), (106), and (193),

$$
\begin{aligned}
\boldsymbol{s}_{4} & =\boldsymbol{s}_{1}+\boldsymbol{s}_{2}+\boldsymbol{s}_{3}=\boldsymbol{h}_{\alpha_{1}}+\left(\boldsymbol{h}_{\alpha_{1}}+\boldsymbol{h}_{\alpha_{3}}\right)+\boldsymbol{h}_{\alpha_{4}} \\
& =\boldsymbol{h}_{\alpha_{3}}+\boldsymbol{h}_{\alpha_{4}} .
\end{aligned}
$$


Hence, $\left\{\alpha_{3}, \alpha_{4}\right\} \in V\left(s_{4}\right)$. Let $\alpha_{5}$ be an integer such that $\alpha_{5} \in \mathcal{I}_{15} \backslash\left\{\alpha_{1}, \ldots, \alpha_{4}\right\}$ and

$$
\boldsymbol{s}_{1}=\boldsymbol{h}_{\alpha_{4}}+\boldsymbol{h}_{\alpha_{5}} .
$$

Then, $\left\{\alpha_{4}, \alpha_{5}\right\} \in V\left(s_{1}\right)$. From (103) and (196),

$$
\boldsymbol{h}_{\alpha_{1}}=\boldsymbol{h}_{\alpha_{4}}+\boldsymbol{h}_{\alpha_{5}} .
$$

From 197) and 193,

$$
\boldsymbol{h}_{\alpha_{1}}+\boldsymbol{h}_{\alpha_{5}}=\boldsymbol{h}_{\alpha_{4}}=\boldsymbol{s}_{3} .
$$

Therefore, $\left\{\alpha_{1}, \alpha_{5}\right\} \in V\left(s_{3}\right)$. From (105) and (196),

$$
\boldsymbol{h}_{\alpha_{2}}+\boldsymbol{h}_{\alpha_{3}}=\boldsymbol{h}_{\alpha_{4}}+\boldsymbol{h}_{\alpha_{5}} \text {. }
$$

From (199) and (195),

$$
\boldsymbol{h}_{\alpha_{2}}+\boldsymbol{h}_{\alpha_{5}}=\boldsymbol{h}_{\alpha_{3}}+\boldsymbol{h}_{\alpha_{4}}=\boldsymbol{s}_{4} .
$$

Hence, $\left\{\alpha_{2}, \alpha_{5}\right\} \in V\left(s_{4}\right)$. Let $\alpha_{6}$ be an integer such that $\alpha_{6} \in \mathcal{I}_{15} \backslash\left\{\alpha_{1}, \ldots, \alpha_{5}\right\}$ and

$$
\boldsymbol{s}_{2}=\boldsymbol{h}_{\alpha_{4}}+\boldsymbol{h}_{\alpha_{6}} .
$$

Then, $\left\{\alpha_{4}, \alpha_{6}\right\} \in V\left(s_{2}\right)$. From (104) and 201,

$$
\boldsymbol{h}_{\alpha_{2}}=\boldsymbol{h}_{\alpha_{4}}+\boldsymbol{h}_{\alpha_{6}} \text {. }
$$

From (202) and 193),

$$
\boldsymbol{h}_{\alpha_{2}}+\boldsymbol{h}_{\alpha_{6}}=\boldsymbol{h}_{\alpha_{4}}=\boldsymbol{s}_{3} .
$$

Therefore, $\left\{\alpha_{2}, \alpha_{6}\right\} \in V\left(s_{3}\right)$. From (106) and (201),

$$
\boldsymbol{h}_{\alpha_{1}}+\boldsymbol{h}_{\alpha_{3}}=\boldsymbol{h}_{\alpha_{4}}+\boldsymbol{h}_{\alpha_{6}} .
$$

From (204) and (195),

$$
\boldsymbol{h}_{\alpha_{1}}+\boldsymbol{h}_{\alpha_{6}}=\boldsymbol{h}_{\alpha_{3}}+\boldsymbol{h}_{\alpha_{4}}=\boldsymbol{s}_{4} .
$$

Hence, $\left\{\alpha_{1}, \alpha_{6}\right\} \in V\left(s_{4}\right)$. Let $\alpha_{7}$ be an integer such that $\alpha_{7} \in \mathcal{I}_{15} \backslash\left\{\alpha_{1}, \ldots, \alpha_{6}\right\}$ and

$$
\boldsymbol{s}_{1}=\boldsymbol{h}_{\alpha_{6}}+\boldsymbol{h}_{\alpha_{7}} \text {. }
$$

Then, $\left\{\alpha_{6}, \alpha_{7}\right\} \in V\left(s_{1}\right)$. From (196) and (206,

$$
\boldsymbol{h}_{\alpha_{4}}+\boldsymbol{h}_{\alpha_{5}}=\boldsymbol{h}_{\alpha_{6}}+\boldsymbol{h}_{\alpha_{7}} .
$$

From 207) and 201,

$$
\boldsymbol{h}_{\alpha_{5}}+\boldsymbol{h}_{\alpha_{7}}=\boldsymbol{h}_{\alpha_{4}}+\boldsymbol{h}_{\alpha_{6}}=\boldsymbol{s}_{2} .
$$

Therefore, $\left\{\alpha_{5}, \alpha_{7}\right\} \in V\left(s_{2}\right)$. From (105) and (206),

$$
\boldsymbol{h}_{\alpha_{2}}+\boldsymbol{h}_{\alpha_{3}}=\boldsymbol{h}_{\alpha_{6}}+\boldsymbol{h}_{\alpha_{7}} \text {. }
$$

From (209) and (203),

$$
\boldsymbol{h}_{\alpha_{3}}+\boldsymbol{h}_{\alpha_{7}}=\boldsymbol{h}_{\alpha_{2}}+\boldsymbol{h}_{\alpha_{6}}=\boldsymbol{s}_{3} .
$$

Hence, $\left\{\alpha_{3}, \alpha_{7}\right\} \in V\left(s_{3}\right)$. From (206), 201), and (193),

$$
\begin{aligned}
\boldsymbol{s}_{4} & =\boldsymbol{s}_{1}+\boldsymbol{s}_{2}+\boldsymbol{s}_{3}=\left(\boldsymbol{h}_{\alpha_{6}}+\boldsymbol{h}_{\alpha_{7}}\right)+\left(\boldsymbol{h}_{\alpha_{4}}+\boldsymbol{h}_{\alpha_{6}}\right)+\boldsymbol{h}_{\alpha_{4}} \\
& =\boldsymbol{h}_{\alpha_{7}} .
\end{aligned}
$$

Therefore, $\left\{\alpha_{7}\right\} \in V\left(s_{4}\right)$. Let $\alpha_{8}$ be an integer such that $\alpha_{8} \in \mathcal{I}_{15} \backslash\left\{\alpha_{1}, \ldots, \alpha_{7}\right\}$, and let $\alpha_{9}$ be an integer such that $\alpha_{9} \in \mathcal{I}_{15} \backslash\left\{\alpha_{1}, \ldots, \alpha_{8}\right\}$ and

$$
\boldsymbol{s}_{1}=\boldsymbol{h}_{\alpha_{8}}+\boldsymbol{h}_{\alpha_{9}} .
$$

Then, $\left\{\alpha_{8}, \alpha_{9}\right\} \in V\left(s_{1}\right)$. Let $\alpha_{10}$ be an integer such that $\alpha_{10} \in \mathcal{I}_{15} \backslash\left\{\alpha_{1}, \ldots, \alpha_{9}\right\}$ and

$$
\boldsymbol{s}_{2}=\boldsymbol{h}_{\alpha_{8}}+\boldsymbol{h}_{\alpha_{10}} .
$$

Then, $\left\{\alpha_{8}, \alpha_{10}\right\} \in V\left(s_{2}\right)$. Let $\alpha_{11}$ be an integer such that $\alpha_{11} \in \mathcal{I}_{15} \backslash\left\{\alpha_{1}, \ldots, \alpha_{10}\right\}$ and

$$
\boldsymbol{s}_{1}=\boldsymbol{h}_{\alpha_{10}}+\boldsymbol{h}_{\alpha_{11}} \text {. }
$$

Then, $\left\{\alpha_{10}, \alpha_{11}\right\} \in V\left(s_{1}\right)$. From (212) and (214),

$$
\boldsymbol{h}_{\alpha_{8}}+\boldsymbol{h}_{\alpha_{9}}=\boldsymbol{h}_{\alpha_{10}}+\boldsymbol{h}_{\alpha_{11}} .
$$

From (215) and (213),

$$
\boldsymbol{h}_{\alpha_{9}}+\boldsymbol{h}_{\alpha_{11}}=\boldsymbol{h}_{\alpha_{8}}+\boldsymbol{h}_{\alpha_{10}}=\boldsymbol{s}_{2} .
$$

Hence, $\left\{\alpha_{9}, \alpha_{11}\right\} \in V\left(s_{2}\right)$. Let $\alpha_{12}$ be an integer such that $\alpha_{12} \in \mathcal{I}_{15} \backslash\left\{\alpha_{1}, \ldots, \alpha_{10}\right\}$ and

$$
\boldsymbol{s}_{3}=\boldsymbol{h}_{\alpha_{8}}+\boldsymbol{h}_{\alpha_{12}} \text {. }
$$

Then, $\left\{\alpha_{8}, \alpha_{12}\right\} \in V\left(s_{3}\right)$. From (217), (212), and (216),

$$
\begin{aligned}
& \boldsymbol{h}_{\alpha_{8}}+\boldsymbol{h}_{\alpha_{12}}=\boldsymbol{s}_{3} \\
\neq \quad & \boldsymbol{s}_{1}+\boldsymbol{s}_{2}=\left(\boldsymbol{h}_{\alpha_{8}}+\boldsymbol{h}_{\alpha_{9}}\right)+\left(\boldsymbol{h}_{\alpha_{9}}+\boldsymbol{h}_{\alpha_{11}}\right) \\
& =\boldsymbol{h}_{\alpha_{8}}+\boldsymbol{h}_{\alpha_{11}} .
\end{aligned}
$$

Therefore, $\alpha_{12} \neq \alpha_{11}$. From (212), (216), and (217),

$$
\begin{aligned}
\boldsymbol{s}_{4} & =\boldsymbol{s}_{1}+\boldsymbol{s}_{2}+\boldsymbol{s}_{3} \\
& =\left(\boldsymbol{h}_{\alpha_{8}}+\boldsymbol{h}_{\alpha_{9}}\right)+\left(\boldsymbol{h}_{\alpha_{9}}+\boldsymbol{h}_{\alpha_{11}}\right)+\left(\boldsymbol{h}_{\alpha_{8}}+\boldsymbol{h}_{\alpha_{12}}\right) \\
& =\boldsymbol{h}_{\alpha_{11}}+\boldsymbol{h}_{\alpha_{12}} .
\end{aligned}
$$

Hence, $\left\{\alpha_{11}, \alpha_{12}\right\} \in V\left(s_{4}\right)$. Let $\alpha_{13}$ be an integer such that $\alpha_{13} \in \mathcal{I}_{15} \backslash\left\{\alpha_{1}, \ldots, \alpha_{12}\right\}$ and

$$
\boldsymbol{s}_{1}=\boldsymbol{h}_{\alpha_{12}}+\boldsymbol{h}_{\alpha_{13}} \text {. }
$$

Then, $\left\{\alpha_{12}, \alpha_{13}\right\} \in V\left(\boldsymbol{s}_{1}\right)$. From (212) and (220),

$$
\boldsymbol{h}_{\alpha_{8}}+\boldsymbol{h}_{\alpha_{9}}=\boldsymbol{h}_{\alpha_{12}}+\boldsymbol{h}_{\alpha_{13}} .
$$

From (221) and (217),

$$
\boldsymbol{h}_{\alpha_{9}}+\boldsymbol{h}_{\alpha_{13}}=\boldsymbol{h}_{\alpha_{8}}+\boldsymbol{h}_{\alpha_{12}}=\boldsymbol{s}_{3} .
$$

Therefore, $\left\{\alpha_{9}, \alpha_{13}\right\} \in V\left(s_{3}\right)$. From (214) and (220),

$$
\boldsymbol{h}_{\alpha_{10}}+\boldsymbol{h}_{\alpha_{11}}=\boldsymbol{h}_{\alpha_{12}}+\boldsymbol{h}_{\alpha_{13}} .
$$

From (223) and (219),

$$
\boldsymbol{h}_{\alpha_{10}}+\boldsymbol{h}_{\alpha_{13}}=\boldsymbol{h}_{\alpha_{11}}+\boldsymbol{h}_{\alpha_{12}}=\boldsymbol{s}_{4} .
$$

Hence, $\left\{\alpha_{10}, \alpha_{13}\right\} \in V\left(s_{4}\right)$. Let $\alpha_{14}$ be an integer such that $\alpha_{14} \in \mathcal{I}_{15} \backslash\left\{\alpha_{1}, \ldots, \alpha_{13}\right\}$ and

$$
\boldsymbol{s}_{2}=\boldsymbol{h}_{\alpha_{12}}+\boldsymbol{h}_{\alpha_{14}} \text {. }
$$


Then, $\left\{\alpha_{12}, \alpha_{14}\right\} \in V\left(s_{2}\right)$. From (213) and (225),

$$
\boldsymbol{h}_{\alpha_{8}}+\boldsymbol{h}_{\alpha_{10}}=\boldsymbol{h}_{\alpha_{12}}+\boldsymbol{h}_{\alpha_{14}} .
$$

From (226) and (217),

$$
\boldsymbol{h}_{\alpha_{10}}+\boldsymbol{h}_{\alpha_{14}}=\boldsymbol{h}_{\alpha_{8}}+\boldsymbol{h}_{\alpha_{12}}=\boldsymbol{s}_{3} .
$$

Therefore, $\left\{\alpha_{10}, \alpha_{14}\right\} \in V\left(s_{3}\right)$. From (216) and (225),

$$
\boldsymbol{h}_{\alpha_{9}}+\boldsymbol{h}_{\alpha_{11}}=\boldsymbol{h}_{\alpha_{12}}+\boldsymbol{h}_{\alpha_{14}} .
$$

From (228) and 219),

$$
\boldsymbol{h}_{\alpha_{9}}+\boldsymbol{h}_{\alpha_{14}}=\boldsymbol{h}_{\alpha_{11}}+\boldsymbol{h}_{\alpha_{12}}=\boldsymbol{s}_{4} .
$$

Hence, $\left\{\alpha_{9}, \alpha_{14}\right\} \in V\left(s_{4}\right)$. Let $\alpha_{15}$ be an integer such that $\alpha_{15} \in \mathcal{I}_{15} \backslash\left\{\alpha_{1}, \ldots, \alpha_{14}\right\}$ and

$$
\boldsymbol{s}_{1}=\boldsymbol{h}_{\alpha_{14}}+\boldsymbol{h}_{\alpha_{15}} \text {. }
$$

Then, $\left\{\alpha_{14}, \alpha_{15}\right\} \in V\left(s_{1}\right)$. From (220) and (230),

$$
\boldsymbol{h}_{\alpha_{12}}+\boldsymbol{h}_{\alpha_{13}}=\boldsymbol{h}_{\alpha_{14}}+\boldsymbol{h}_{\alpha_{15}} .
$$

From 231) and 225],

$$
\boldsymbol{h}_{\alpha_{13}}+\boldsymbol{h}_{\alpha_{15}}=\boldsymbol{h}_{\alpha_{12}}+\boldsymbol{h}_{\alpha_{14}}=\boldsymbol{s}_{2} .
$$

Therefore, $\left\{\alpha_{13}, \alpha_{15}\right\} \in V\left(s_{2}\right)$. From (214) and (230),

$$
\boldsymbol{h}_{\alpha_{10}}+\boldsymbol{h}_{\alpha_{11}}=\boldsymbol{h}_{\alpha_{14}}+\boldsymbol{h}_{\alpha_{15}} .
$$

From (233) and (227),

$$
\boldsymbol{h}_{\alpha_{11}}+\boldsymbol{h}_{\alpha_{15}}=\boldsymbol{h}_{\alpha_{10}}+\boldsymbol{h}_{\alpha_{14}}=\boldsymbol{s}_{3} .
$$

Hence, $\left\{\alpha_{11}, \alpha_{15}\right\} \in V\left(s_{3}\right)$. From (212) and (230),

$$
\boldsymbol{h}_{\alpha_{8}}+\boldsymbol{h}_{\alpha_{9}}=\boldsymbol{h}_{\alpha_{14}}+\boldsymbol{h}_{\alpha_{15}} .
$$

From (235) and 229),

$$
\boldsymbol{h}_{\alpha_{8}}+\boldsymbol{h}_{\alpha_{15}}=\boldsymbol{h}_{\alpha_{9}}+\boldsymbol{h}_{\alpha_{14}}=\boldsymbol{s}_{4} .
$$

Therefore, $\left\{\alpha_{8}, \alpha_{15}\right\} \in V\left(s_{4}\right)$. Let $\sigma$ be the permutation of $\mathcal{I}_{15}$ such that $\sigma(j)=\alpha_{j}$ for each $j \in \mathcal{I}_{15}$. Then, $\sigma$ satisfies (99), (100), (101), and (102).

\section{B. Construction of $[7,3,4]$ P-RIO Code}

Let $C$ be a $(7,4)$ Hamming code.

Example 3: The parity check matrix $H$ of $C$ is as follows.

$$
H=\left(\begin{array}{lllllll}
1 & 0 & 1 & 0 & 1 & 0 & 1 \\
0 & 1 & 1 & 0 & 0 & 1 & 1 \\
0 & 0 & 0 & 1 & 1 & 1 & 1
\end{array}\right)
$$

For each $s \in\{0,1\}^{3} \backslash\{000\}, V(s)$ is as follows [5].

$$
\begin{aligned}
& V(100)=\{\{1\},\{2,3\},\{4,5\},\{6,7\}\}, \\
& V(010)=\{\{2\},\{1,3\},\{4,6\},\{5,7\}\}, \\
& V(110)=\{\{3\},\{1,2\},\{4,7\},\{5,6\}\}, \\
& V(001)=\{\{4\},\{1,5\},\{2,6\},\{3,7\}\}, \\
& V(101)=\{\{5\},\{1,4\},\{2,7\},\{3,6\}\}, \\
& V(011)=\{\{6\},\{1,7\},\{2,4\},\{3,5\}\}, \\
& V(111)=\{\{7\},\{1,6\},\{2,5\},\{3,4\}\} .
\end{aligned}
$$

For some $s_{1}, \ldots, s_{4} \in\{0,1\}^{3}$, we obtain $\boldsymbol{x}_{1}, \ldots, \boldsymbol{x}_{4} \in$ $\{0,1\}^{7}$ that satisfy the conditions of Theorem 2

Case : $\boldsymbol{s}_{1}=111, \boldsymbol{s}_{2}=100, \boldsymbol{s}_{3}=110$, and $\boldsymbol{s}_{4}=101$.

Let $I\left(\boldsymbol{x}_{1}\right)=\{7\}, I\left(\boldsymbol{x}_{2}\right)=\{1\}, I\left(\boldsymbol{x}_{3}\right)=\{3\}$, and $I\left(\boldsymbol{x}_{4}\right)=\{5\}$. Therefore, $\boldsymbol{x}_{1}=0000001, \boldsymbol{x}_{2}=1000000, \boldsymbol{x}_{3}=$ 0010000 , and $\boldsymbol{x}_{4}=0000100$.

Case : $s_{1}=001, s_{2}=110, s_{3}=100$, and $s_{4}=001$.

Let $I\left(\boldsymbol{x}_{1}\right)=\{4\}, I\left(\boldsymbol{x}_{2}\right)=\{3\}, I\left(\boldsymbol{x}_{3}\right)=\{1\}$, and $I\left(\boldsymbol{x}_{4}\right)=$ $\{2,6\}$. Therefore, $\boldsymbol{x}_{1}=0001000, \boldsymbol{x}_{2}=0010000, \boldsymbol{x}_{3}=$ 1000000 , and $\boldsymbol{x}_{4}=0100010$.

Case : $s_{1}=010, s_{2}=101, s_{3}=010$, and $s_{4}=101$.

Let $I\left(\boldsymbol{x}_{1}\right)=\{2\}, I\left(\boldsymbol{x}_{2}\right)=\{1,4\}, I\left(\boldsymbol{x}_{3}\right)=\{5,7\}$, and $I\left(\boldsymbol{x}_{4}\right)=\{3,6\}$. Therefore, $\boldsymbol{x}_{1}=0100000, \boldsymbol{x}_{2}=$ $1001000, \boldsymbol{x}_{3}=0000101$, and $\boldsymbol{x}_{4}=0010010$.

For any $\boldsymbol{s}_{1}, \boldsymbol{s}_{2}, \boldsymbol{s}_{3}, \boldsymbol{s}_{4} \in\{0,1\}^{3}$, we have $\boldsymbol{x}_{1}, \boldsymbol{x}_{2}, \boldsymbol{x}_{3}, \boldsymbol{x}_{4} \in$ $\{0,1\}^{7}$, which satisfy the conditions of Theorem 2 as described below.

We define $I_{0}=\left\{i \mid i \in \mathcal{I}_{4}, \boldsymbol{s}_{i}=000\right\}$. For any $\boldsymbol{s}_{i} \neq 000$, let $j_{i}$ be an integer such that $j_{i} \in \mathcal{I}_{7}$ and $\boldsymbol{s}_{i}=\boldsymbol{h}_{j_{i}}$.

Case 1: $I_{0} \neq \emptyset$.

Let $t^{\prime}=4-\left|I_{0}\right|$. For any $\boldsymbol{s}_{k_{1}}, \ldots, \boldsymbol{s}_{k_{t^{\prime}}}\left(\left\{k_{1}, \ldots, k_{t^{\prime}}\right\}=\mathcal{I}_{4} \backslash\right.$ $I_{0}$ ), we use one of the following cases to obtain $\boldsymbol{x}_{k_{1}}, \ldots, \boldsymbol{x}_{k_{t^{\prime}}}$ that satisfy the conditions of Theorem 2 Further, for every $i \in I_{0}$, let $\boldsymbol{x}_{i}=0000000$.

In the following, we assume $I_{0}=\emptyset$.

Case 2: $s_{i} \neq s_{i^{\prime}}$ for any $i, i^{\prime} \in \mathcal{I}_{4}, i \neq i^{\prime}$.

Let $I\left(\boldsymbol{x}_{i}\right)=\left\{j_{i}\right\}$ for each $i \in \mathcal{I}_{4}$.

Case 3: There exist $2 \leq m \leq 4$ and the permutation $\pi$ of $\mathcal{I}_{4}$ such that $\boldsymbol{s}_{\pi(1)}=\cdots=\boldsymbol{s}_{\pi(m)}$ and $\boldsymbol{s}_{\pi(i)} \neq \boldsymbol{s}_{\pi\left(i^{\prime}\right)}$ for any $i, i^{\prime} \in\{1, m+1, \ldots, 4\}, i \neq i^{\prime}$.

Let $I\left(\boldsymbol{x}_{\pi(1)}\right)=\left\{j_{\pi(1)}\right\}, I\left(\boldsymbol{x}_{\pi(m+1)}\right)=\left\{j_{\pi(m+1)}\right\}, \ldots$, $I\left(\boldsymbol{x}_{\pi(4)}\right)=\left\{j_{\pi(4)}\right\}$. From Theorem [3] we have the permutation $\sigma$ of $\mathcal{I}_{7}$ such that

$$
\begin{aligned}
V\left(\boldsymbol{s}_{\pi(2)}\right)= & \{\{\sigma(1)\},\{\sigma(2), \sigma(3)\}, \\
& \{\sigma(4), \sigma(5)\},\{\sigma(6), \sigma(7)\}\} .
\end{aligned}
$$

Clearly, $\sigma(1)=j_{\pi(1)}$. Hence, there are a minimum of $(m-$ 1) elements $\alpha$ in $\{2,4,6\}$ such that $\left\{j_{\pi(m+1)}, \ldots, j_{\pi(4)}\right\} \cap$ $\{\sigma(\alpha), \sigma(\alpha+1)\}=\emptyset$. We denote these $\alpha$ by $\alpha_{1}, \ldots, \alpha_{m-1}$. Then, let $I\left(\boldsymbol{x}_{\pi(2)}\right)=\left\{\sigma\left(\alpha_{1}\right), \sigma\left(\alpha_{1}+1\right)\right\}, \ldots, I\left(\boldsymbol{x}_{\pi(m)}\right)=$ $\left\{\sigma\left(\alpha_{m-1}\right), \sigma\left(\alpha_{m-1}+1\right)\right\}$.

Case 4: There exists the permutation $\pi$ of $\mathcal{I}_{4}$ such that $s_{\pi(1)}=$ $\boldsymbol{s}_{\pi(2)}, \boldsymbol{s}_{\pi(3)}=\boldsymbol{s}_{\pi(4)}$, and $\boldsymbol{s}_{\pi(1)} \neq \boldsymbol{s}_{\pi(3)}$.

From Theorem 4, we have the permutation $\sigma$ of $\mathcal{I}_{7}$ such that

$$
\begin{aligned}
V\left(\boldsymbol{s}_{\pi(1)}\right)= & \{\{\sigma(1)\},\{\sigma(2), \sigma(3)\}, \\
& \{\sigma(4), \sigma(5)\},\{\sigma(6), \sigma(7)\}\}, \\
V\left(\boldsymbol{s}_{\pi(3)}\right)= & \{\{\sigma(2)\},\{\sigma(1), \sigma(3)\}, \\
& \{\sigma(4), \sigma(6)\},\{\sigma(5), \sigma(7)\}\} .
\end{aligned}
$$

Hence, let $I\left(\boldsymbol{x}_{\pi(1)}\right)=\{\sigma(1)\}, I\left(\boldsymbol{x}_{\pi(2)}\right)=\{\sigma(2), \sigma(3)\}$, $I\left(\boldsymbol{x}_{\pi(3)}\right)=\{\sigma(4), \sigma(6)\}$, and $I\left(\boldsymbol{x}_{\pi(4)}\right)=\{\sigma(5), \sigma(7)\}$.

Therefore, from Theorem 2 the [7,3,4] P-RIO code can be constructed. This code stores more pages than the $[7,3,3]$ RIO code, which is constructed using the same $(7,4)$ Hamming code. 


\section{Construction of $[15,4,8]$ P-RIO Code}

Let $C$ be a $(15,11)$ Hamming code. For any $s_{1}, \ldots, s_{8} \in$ $\{0,1\}^{4}$, we have $\boldsymbol{x}_{1}, \ldots, \boldsymbol{x}_{8} \in\{0,1\}^{15}$, which satisfy the conditions of Theorem 2 as follows.

We define $I_{0}=\left\{i \mid i \in \mathcal{I}_{8}, \boldsymbol{s}_{i}=0000\right\}$. For any $\boldsymbol{s}_{i} \neq 0000$, let $j_{i}$ be an integer such that $j_{i} \in \mathcal{I}_{15}$ and $\boldsymbol{s}_{i}=\boldsymbol{h}_{j_{i}}$.

Case 1: $I_{0} \neq \emptyset$.

We have $\boldsymbol{x}_{1}, \ldots, \boldsymbol{x}_{8}$ as for the case of the $[7,3,4]$ P-RIO code.

Case 2: $\boldsymbol{s}_{i} \neq \boldsymbol{s}_{i^{\prime}}$ for any $i, i^{\prime} \in \mathcal{I}_{8}, i \neq i^{\prime}$.

Let $I\left(\boldsymbol{x}_{i}\right)=\left\{j_{i}\right\}$ for each $i \in \mathcal{I}_{8}$.

Case 3: There exist $2 \leq m \leq 8$ and the permutation $\pi$ of $\mathcal{I}_{8}$ such that $\boldsymbol{s}_{\pi(1)}=\cdots=\boldsymbol{s}_{\pi(m)}$ and $\boldsymbol{s}_{\pi(i)} \neq \boldsymbol{s}_{\pi\left(i^{\prime}\right)}$ for any $i, i^{\prime} \in\{1, m+1, \ldots, 8\}, i \neq i^{\prime}$.

Let $I\left(\boldsymbol{x}_{\pi(1)}\right)=\left\{j_{\pi(1)}\right\}, I\left(\boldsymbol{x}_{\pi(m+1)}\right)=\left\{j_{\pi(m+1)}\right\}, \ldots$, $I\left(\boldsymbol{x}_{\pi(8)}\right)=\left\{j_{\pi(8)}\right\}$. From Theorem [3, we have the permutation $\sigma$ of $\mathcal{I}_{15}$ such that

$$
\begin{aligned}
V\left(\boldsymbol{s}_{\pi(2)}\right)= & \{\{\sigma(1)\},\{\sigma(2), \sigma(3)\},\{\sigma(4), \sigma(5)\}, \\
& \{\sigma(6), \sigma(7)\},\{\sigma(8), \sigma(9)\},\{\sigma(10), \sigma(11)\}, \\
& \{\sigma(12), \sigma(13)\},\{\sigma(14), \sigma(15)\}\} .
\end{aligned}
$$

Clearly, $\sigma(1)=j_{\pi(1)}$. Hence, there are a minimum of $(m-1)$ elements $\alpha$ in $\{2,4,6,8,10,12,14\}$, such that $\left\{j_{\pi(m+1)}, \ldots, j_{\pi(8)}\right\} \cap\{\sigma(\alpha), \sigma(\alpha+1)\}=\emptyset$. We denote these $\alpha$ by $\alpha_{1}, \ldots, \alpha_{m-1}$. Then, let $I\left(\boldsymbol{x}_{\pi(2)}\right)=\left\{\sigma\left(\alpha_{1}\right), \sigma\left(\alpha_{1}+1\right)\right\}$, $\ldots, I\left(\boldsymbol{x}_{\pi(m)}\right)=\left\{\sigma\left(\alpha_{m-1}\right), \sigma\left(\alpha_{m-1}+1\right)\right\}$.

Case 4: There exist $2 \leq m_{1}, m_{2} \leq 6$, where $m_{1} \geq m_{2}$, and the permutation $\pi$ of $\mathcal{I}_{8}$ such that $s_{\pi(1)}=\cdots=s_{\pi\left(m_{1}\right)}$, $\boldsymbol{s}_{\pi\left(m_{1}+1\right)}=\cdots=\boldsymbol{s}_{\pi\left(m_{1}+m_{2}\right)}$, and $\boldsymbol{s}_{\pi(i)} \neq \boldsymbol{s}_{\pi\left(i^{\prime}\right)}$ for any $i, i^{\prime} \in\left\{1, m_{1}+1, m_{1}+m_{2}+1, \ldots, 8\right\}, i \neq i^{\prime}$.

Let $I\left(\boldsymbol{x}_{\pi\left(m_{1}+m_{2}+1\right)}\right)=\left\{j_{\pi\left(m_{1}+m_{2}+1\right)}\right\}, \ldots, I\left(\boldsymbol{x}_{\pi(8)}\right)=$ $\left\{j_{\pi(8)}\right\}$. From Theorem 4 we have the permutation $\sigma$ of $\mathcal{I}_{15}$ such that

$$
\begin{aligned}
& V\left(s_{\pi(1)}\right) \\
= & \{\{\sigma(1)\},\{\sigma(2), \sigma(3)\},\{\sigma(4), \sigma(5)\}, \\
& \{\sigma(6), \sigma(7)\},\{\sigma(8), \sigma(9)\},\{\sigma(10), \sigma(11)\}, \\
& \{\sigma(12), \sigma(13)\},\{\sigma(14), \sigma(15)\}\}, \\
& V\left(s_{\pi\left(m_{1}+1\right)}\right) \\
= & \{\{\sigma(2)\},\{\sigma(1), \sigma(3)\},\{\sigma(4), \sigma(6)\}, \\
& \{\sigma(5), \sigma(7)\},\{\sigma(8), \sigma(10)\},\{\sigma(9), \sigma(11)\}, \\
& \{\sigma(12), \sigma(14)\},\{\sigma(13), \sigma(15)\}\} .
\end{aligned}
$$

Clearly, $\left\{j_{\pi\left(m_{1}+m_{2}+1\right)}, \ldots, j_{\pi(8)}\right\} \cap\{\sigma(1), \sigma(2)\}=\emptyset$. We define $A_{1}=\left\{j_{\pi(i)} \mid i \in\left\{m_{1}+m_{2}+1, \ldots, 8\right\}, j_{\pi(i)} \in\right.$ $\{\sigma(3), \ldots, \sigma(7)\}\}, A_{2}=\left\{j_{\pi(i)} \mid i \in\left\{m_{1}+m_{2}+\right.\right.$ $\left.1, \ldots, 8\}, j_{\pi(i)} \in\{\sigma(8), \ldots, \sigma(15)\}\right\}, a_{1}=\left|A_{1}\right|, a_{2}=\left|A_{2}\right|$. Then, $a_{1}+a_{2}=8-m_{1}-m_{2}$.

Case 4-1: $m_{1}=m_{2}=2$.

Case 4-1-1: $a_{1}=0$ and $a_{2}=4$.

Let $I\left(\boldsymbol{x}_{\pi(1)}\right)=\{\sigma(1)\}, I\left(\boldsymbol{x}_{\pi(2)}\right)=\{\sigma(2), \sigma(3)\}$, $I\left(\boldsymbol{x}_{\pi(3)}\right)=\{\sigma(4), \sigma(6)\}, I\left(\boldsymbol{x}_{\pi(4)}\right)=\{\sigma(5), \sigma(7)\}$.

Case 4-1-2: $a_{1}=1$ and $a_{2}=3$.
Let $I\left(\boldsymbol{x}_{\pi(1)}\right)=\{\sigma(1)\}$ and $I\left(\boldsymbol{x}_{\pi(2)}\right)=\{\sigma(\alpha), \sigma(\alpha+1)\}$ such that $\alpha \in\{4,6\}$ and $A_{1} \cap\{\sigma(\alpha), \sigma(\alpha+1)\}=\emptyset$. Let $I\left(\boldsymbol{x}_{\pi(3)}\right)=\{\sigma(2)\}$ and $I\left(\boldsymbol{x}_{\pi(4)}\right)=\{\sigma(\beta), \sigma(\beta+2)\}$ such that $\beta \in\{8,9,12,13\}$ and $A_{2} \cap\{\sigma(\beta), \sigma(\beta+2)\}=\emptyset$.

Case 4-1-3: $a_{1}=2$ and $a_{2}=2$.

Let $I\left(\boldsymbol{x}_{\pi(1)}\right)=\{\sigma(1)\}$ and $I\left(\boldsymbol{x}_{\pi(2)}\right)=\{\sigma(\alpha), \sigma(\alpha+1)\}$ such that $\alpha \in\{2,4,6\}$ and $A_{1} \cap\{\sigma(\alpha), \sigma(\alpha+1)\}=$ $\emptyset$. Let $I\left(\boldsymbol{x}_{\pi(3)}\right)=\left\{\sigma\left(\beta_{1}\right), \sigma\left(\beta_{1}+2\right)\right\}$ and $I\left(\boldsymbol{x}_{\pi(4)}\right)=$ $\left\{\sigma\left(\beta_{2}\right), \sigma\left(\beta_{2}+2\right)\right\}$ such that $\beta_{1}, \beta_{2} \in\{8,9,12,13\}, \beta_{1} \neq \beta_{2}$, and $A_{2} \cap\left\{\sigma\left(\beta_{1}\right), \sigma\left(\beta_{1}+2\right), \sigma\left(\beta_{2}\right), \sigma\left(\beta_{2}+2\right)\right\}=\emptyset$.

Case 4-1-4: $a_{1}=3$ and $a_{2}=1$.

Let $I\left(\boldsymbol{x}_{\pi(1)}\right)=\{\sigma(1)\}$ and $I\left(\boldsymbol{x}_{\pi(2)}\right)=\{\sigma(\alpha), \sigma(\alpha+1)\}$ such that $\alpha \in\{8,10\}$ and $A_{2} \cap\{\sigma(\alpha), \sigma(\alpha+1)\}=\emptyset$. Let $I\left(\boldsymbol{x}_{\pi(3)}\right)=\{\sigma(2)\}$ and $I\left(\boldsymbol{x}_{\pi(4)}\right)=\{\sigma(\beta), \sigma(\beta+2)\}$ such that $\beta \in\{12,13\}$ and $A_{2} \cap\{\sigma(\beta), \sigma(\beta+2)\}=\emptyset$.

Case 4-1-5: $a_{1}=4$ and $a_{2}=0$.

Let $I\left(\boldsymbol{x}_{\pi(1)}\right)=\{\sigma(1)\}, I\left(\boldsymbol{x}_{\pi(2)}\right)=\{\sigma(8), \sigma(9)\}$, $I\left(\boldsymbol{x}_{\pi(3)}\right)=\{\sigma(2)\}$, and $I\left(\boldsymbol{x}_{\pi(4)}\right)=\{\sigma(12), \sigma(14)\}$.

Case 4-2: $m_{1}=3$ and $m_{2}=2$.

Case 4-2-1: $a_{1}=0$ and $a_{2}=3$.

Let $I\left(\boldsymbol{x}_{\pi(1)}\right)=\{\sigma(1)\}, I\left(\boldsymbol{x}_{\pi(2)}\right)=\{\sigma(4), \sigma(5)\}$, and $I\left(\boldsymbol{x}_{\pi(3)}\right)=\{\sigma(6), \sigma(7)\}$. Let $I\left(\boldsymbol{x}_{\pi(4)}\right)=\{\sigma(2)\}$ and $I\left(\boldsymbol{x}_{\pi(5)}\right)=\{\sigma(\alpha), \sigma(\alpha+2)\}$ such that $\alpha \in\{8,9,12,13\}$ and $A_{2} \cap\{\sigma(\alpha), \sigma(\alpha+2)\}=\emptyset$.

Case 4-2-2: $a_{1}=1$ and $a_{2}=2$.

Let $I\left(\boldsymbol{x}_{\pi(1)}\right)=\{\sigma(1)\}, I\left(\boldsymbol{x}_{\pi(2)}\right)=\left\{\sigma\left(\alpha_{1}\right), \sigma\left(\alpha_{1}+1\right)\right\}$, and $I\left(\boldsymbol{x}_{\pi(3)}\right)=\left\{\sigma\left(\alpha_{2}\right), \sigma\left(\alpha_{2}+1\right)\right\}$ such that $\alpha_{1}, \alpha_{2} \in$ $\{2,4,6\}, \alpha_{1} \neq \alpha_{2}$, and $A_{1} \cap\left\{\sigma\left(\alpha_{1}\right), \sigma\left(\alpha_{1}+1\right), \sigma\left(\alpha_{2}\right), \sigma\left(\alpha_{2}+\right.\right.$ $1)\}=\emptyset$. Let $I\left(\boldsymbol{x}_{\pi(4)}\right)=\left\{\sigma\left(\beta_{1}\right), \sigma\left(\beta_{1}+2\right)\right\}$ and $I\left(\boldsymbol{x}_{\pi(5)}\right)=$ $\left\{\sigma\left(\beta_{2}\right), \sigma\left(\beta_{2}+2\right)\right\}$ such that $\beta_{1}, \beta_{2} \in\{8,9,12,13\}, \beta_{1} \neq \beta_{2}$, and $A_{2} \cap\left\{\sigma\left(\beta_{1}\right), \sigma\left(\beta_{1}+2\right), \sigma\left(\beta_{2}\right), \sigma\left(\beta_{2}+2\right)\right\}=\emptyset$.

Case 4-2-3: $a_{1}=2$ and $a_{2}=1$.

Let $I\left(\boldsymbol{x}_{\pi(1)}\right)=\{\sigma(1)\}, I\left(\boldsymbol{x}_{\pi(2)}\right)=\{\sigma(\alpha), \sigma(\alpha+1)\}$, and $I\left(\boldsymbol{x}_{\pi(3)}\right)=\{\sigma(\alpha+2), \sigma(\alpha+3)\}$ such that $\alpha \in\{8,12\}$ and $A_{2} \cap\{\sigma(\alpha), \sigma(\alpha+1), \sigma(\alpha+2), \sigma(\alpha+3)\}=\emptyset$. Let $I\left(\boldsymbol{x}_{\pi(4)}\right)=\{\sigma(2)\}$ and $I\left(\boldsymbol{x}_{\pi(5)}\right)=\{\sigma(\beta), \sigma(\beta+2)\}$ such that $\beta \in\{8,9,12,13\}$ and $\left(A_{2} \cup\{\sigma(\alpha), \sigma(\alpha+1), \sigma(\alpha+\right.$ $2), \sigma(\alpha+3)\}) \cap\{\sigma(\beta), \sigma(\beta+2)\}=\emptyset$.

Case 4-2-4: $a_{1}=3$ and $a_{2}=0$.

Let $I\left(\boldsymbol{x}_{\pi(1)}\right)=\{\sigma(1)\}, I\left(\boldsymbol{x}_{\pi(2)}\right)=\{\sigma(8), \sigma(9)\}$, $I\left(\boldsymbol{x}_{\pi(3)}\right)=\{\sigma(10), \sigma(11)\}, I\left(\boldsymbol{x}_{\pi(4)}\right)=\{\sigma(2)\}$, and $I\left(\boldsymbol{x}_{\pi(5)}\right)=\{\sigma(12), \sigma(14)\}$.

Case 4-3: $m_{1}=m_{2}=3$.

Case 4-3-1: $a_{1}=0$ and $a_{2}=2$.

Let $I\left(\boldsymbol{x}_{\pi(1)}\right)=\{\sigma(1)\}, I\left(\boldsymbol{x}_{\pi(2)}\right)=\{\sigma(4), \sigma(5)\}$, and $I\left(\boldsymbol{x}_{\pi(3)}\right)=\{\sigma(6), \sigma(7)\}$. Let $I\left(\boldsymbol{x}_{\pi(4)}\right)=\{\sigma(2)\}, I\left(\boldsymbol{x}_{\pi(5)}\right)=$ $\left\{\sigma\left(\alpha_{1}\right), \sigma\left(\alpha_{1}+2\right)\right\}$, and $I\left(\boldsymbol{x}_{\pi(6)}\right)=\left\{\sigma\left(\alpha_{2}\right), \sigma\left(\alpha_{2}+2\right)\right\}$ such that $\alpha_{1}, \alpha_{2} \in\{8,9,12,13\}, \alpha_{1} \neq \alpha_{2}$, and $A_{2} \cap\left\{\sigma\left(\alpha_{1}\right), \sigma\left(\alpha_{1}+\right.\right.$ 2), $\left.\sigma\left(\alpha_{2}\right), \sigma\left(\alpha_{2}+2\right)\right\}=\emptyset$.

Case 4-3-2: $a_{1}=a_{2}=1$.

Let $I\left(\boldsymbol{x}_{\pi(1)}\right)=\{\sigma(1)\}, I\left(\boldsymbol{x}_{\pi(2)}\right)=\left\{\sigma\left(\alpha_{1}\right), \sigma\left(\alpha_{1}+1\right)\right\}$, and $I\left(\boldsymbol{x}_{\pi(3)}\right)=\left\{\sigma\left(\alpha_{2}\right), \sigma\left(\alpha_{2}+1\right)\right\}$ such that $\alpha_{1}, \alpha_{2} \in$ $\{2,4,6\}, \alpha_{1} \neq \alpha_{2}$, and $A_{1} \cap\left\{\sigma\left(\alpha_{1}\right), \sigma\left(\alpha_{1}+1\right), \sigma\left(\alpha_{2}\right), \sigma\left(\alpha_{2}+\right.\right.$ $1)\}=\emptyset$. Let $I\left(\boldsymbol{x}_{\pi(4)}\right)=\left\{\sigma\left(\beta_{1}\right), \sigma\left(\beta_{1}+2\right)\right\}, I\left(\boldsymbol{x}_{\pi(5)}\right)=$ $\left\{\sigma\left(\beta_{2}\right), \sigma\left(\beta_{2}+2\right)\right\}$, and $I\left(\boldsymbol{x}_{\pi(6)}\right)=\left\{\sigma\left(\beta_{3}\right), \sigma\left(\beta_{3}+2\right)\right\}$ such that $\beta_{1}, \beta_{2}, \beta_{3} \in\{8,9,12,13\}, \beta_{1} \neq \beta_{2}, \beta_{1} \neq \beta_{3}, \beta_{2} \neq \beta_{3}$, 
and $A_{2} \cap\left\{\sigma\left(\beta_{1}\right), \sigma\left(\beta_{1}+2\right), \sigma\left(\beta_{2}\right), \sigma\left(\beta_{2}+2\right), \sigma\left(\beta_{3}\right), \sigma\left(\beta_{3}+\right.\right.$ 2) $\}=\emptyset$.

Case 4-3-3: $a_{1}=2$ and $a_{2}=0$.

Let $I\left(\boldsymbol{x}_{\pi(1)}\right)=\{\sigma(1)\}, I\left(\boldsymbol{x}_{\pi(2)}\right)=\{\sigma(8), \sigma(9)\}$, $I\left(\boldsymbol{x}_{\pi(3)}\right)=\{\sigma(10), \sigma(11)\}, I\left(\boldsymbol{x}_{\pi(4)}\right)=\{\sigma(2)\}, I\left(\boldsymbol{x}_{\pi(5)}\right)=$ $\{\sigma(12), \sigma(14)\}$, and $I\left(\boldsymbol{x}_{\pi(6)}\right)=\{\sigma(13), \sigma(15)\}$.

Case 4-4: $m_{1}=4$ and $m_{2}=2$.

Case 4-4-1: $a_{1}=0$ and $a_{2}=2$.

Let $I\left(\boldsymbol{x}_{\pi(1)}\right)=\{\sigma(1)\}, I\left(\boldsymbol{x}_{\pi(2)}\right)=\{\sigma(2), \sigma(3)\}$, $I\left(\boldsymbol{x}_{\pi(3)}\right)=\{\sigma(4), \sigma(5)\}$, and $I\left(\boldsymbol{x}_{\pi(4)}\right)=\{\sigma(6), \sigma(7)\}$. Let $I\left(\boldsymbol{x}_{\pi(5)}\right)=\left\{\sigma\left(\alpha_{1}\right), \sigma\left(\alpha_{1}+2\right)\right\}$ and $I\left(\boldsymbol{x}_{\pi(6)}\right)=$ $\left\{\sigma\left(\alpha_{2}\right), \sigma\left(\alpha_{2}+2\right)\right\}$ such that $\alpha_{1}, \alpha_{2} \in\{8,9,12,13\}, \alpha_{1} \neq \alpha_{2}$, and $A_{2} \cap\left\{\sigma\left(\alpha_{1}\right), \sigma\left(\alpha_{1}+2\right), \sigma\left(\alpha_{2}\right), \sigma\left(\alpha_{2}+2\right)\right\}=\emptyset$.

Case 4-4-2: $a_{1}=a_{2}=1$.

Let $I\left(\boldsymbol{x}_{\pi(1)}\right)=\{\sigma(1)\}$ and $I\left(\boldsymbol{x}_{\pi(2)}\right)=\{\sigma(\alpha), \sigma(\alpha+1)\}$ such that $\alpha \in\{4,6\}$ and $A_{1} \cap\{\sigma(\alpha), \sigma(\alpha+1)\}=\emptyset$. Let $I\left(\boldsymbol{x}_{\pi(3)}\right)=\{\sigma(\beta), \sigma(\beta+1)\}$ and $I\left(\boldsymbol{x}_{\pi(4)}\right)=\{\sigma(\beta+$ $2), \sigma(\beta+3)\}$ such that $\beta \in\{8,12\}$ and $A_{2} \cap\{\sigma(\beta), \sigma(\beta+$ $1), \sigma(\beta+2), \sigma(\beta+3)\}=\emptyset$. Let $I\left(\boldsymbol{x}_{\pi(5)}\right)=\{\sigma(2)\}$ and $I\left(\boldsymbol{x}_{\pi(6)}\right)=\{\sigma(\gamma), \sigma(\gamma+2)\}$ such that $\gamma \in\{8,9,12,13\}$ and $\left(A_{2} \cup\{\sigma(\beta), \sigma(\beta+1), \sigma(\beta+2), \sigma(\beta+3)\}\right) \cap\{\sigma(\gamma), \sigma(\gamma+2)\}=$ $\emptyset$.

Case 4-4-3: $a_{1}=2$ and $a_{2}=0$.

Let $I\left(\boldsymbol{x}_{\pi(1)}\right)=\{\sigma(1)\}, I\left(\boldsymbol{x}_{\pi(2)}\right)=\{\sigma(8), \sigma(9)\}$, $I\left(\boldsymbol{x}_{\pi(3)}\right)=\{\sigma(10), \sigma(11)\}$, and $I\left(\boldsymbol{x}_{\pi(4)}\right)=\{\sigma(\alpha), \sigma(\alpha+1)\}$ such that $\alpha \in\{2,4,6\}$ and $A_{1} \cap\{\sigma(\alpha), \sigma(\alpha+1)\}=\emptyset$. Let $I\left(\boldsymbol{x}_{\pi(5)}\right)=\{\sigma(12), \sigma(14)\}$ and $I\left(\boldsymbol{x}_{\pi(6)}\right)=\{\sigma(13), \sigma(15)\}$.

Case 4-5: $m_{1}=4$ and $m_{2}=3$.

Case 4-5-1: $a_{1}=0$ and $a_{2}=1$.

Let $I\left(\boldsymbol{x}_{\pi(1)}\right)=\{\sigma(1)\}, I\left(\boldsymbol{x}_{\pi(2)}\right)=\{\sigma(2), \sigma(3)\}$, $I\left(\boldsymbol{x}_{\pi(3)}\right)=\{\sigma(4), \sigma(5)\}$, and $I\left(\boldsymbol{x}_{\pi(4)}\right)=\{\sigma(6), \sigma(7)\}$. Let $I\left(\boldsymbol{x}_{\pi(5)}\right)=\left\{\sigma\left(\alpha_{1}\right), \sigma\left(\alpha_{1}+2\right)\right\}, I\left(\boldsymbol{x}_{\pi(6)}\right)=\left\{\sigma\left(\alpha_{2}\right), \sigma\left(\alpha_{2}+\right.\right.$ $2)\}$, and $I\left(\boldsymbol{x}_{\pi(7)}\right)=\left\{\sigma\left(\alpha_{3}\right), \sigma\left(\alpha_{3}+2\right)\right\}$ such that $\alpha_{1}, \alpha_{2}, \alpha_{3} \in$ $\{8,9,12,13\}, \alpha_{1} \neq \alpha_{2}, \alpha_{1} \neq \alpha_{3}, \alpha_{2} \neq \alpha_{3}$, and $A_{2} \cap$ $\left\{\sigma\left(\alpha_{1}\right), \sigma\left(\alpha_{1}+2\right), \sigma\left(\alpha_{2}\right), \sigma\left(\alpha_{2}+2\right), \sigma\left(\alpha_{3}\right), \sigma\left(\alpha_{3}+2\right)\right\}=\emptyset$. Case 4-5-2: $a_{1}=1$ and $a_{2}=0$.

Let $I\left(\boldsymbol{x}_{\pi(1)}\right)=\{\sigma(8), \sigma(9)\}, I\left(\boldsymbol{x}_{\pi(2)}\right)=\{\sigma(10), \sigma(11)\}$, $I\left(\boldsymbol{x}_{\pi(3)}\right)=\{\sigma(12), \sigma(13)\}$, and $I\left(\boldsymbol{x}_{\pi(4)}\right)=\{\sigma(14), \sigma(15)\}$. Let $I\left(\boldsymbol{x}_{\pi(5)}\right)=\{\sigma(2)\}, I\left(\boldsymbol{x}_{\pi(6)}\right)=\left\{\sigma\left(\alpha_{1}\right), \sigma\left(\alpha_{1}+2\right)\right\}$, and $I\left(\boldsymbol{x}_{\pi(7)}\right)=\left\{\sigma\left(\alpha_{2}\right), \sigma\left(\alpha_{2}+2\right)\right\}$ such that $\alpha_{1}, \alpha_{2} \in$ $\{1,4,5\}, \alpha_{1} \neq \alpha_{2}$, and $A_{1} \cap\left\{\sigma\left(\alpha_{1}\right), \sigma\left(\alpha_{1}+2\right), \sigma\left(\alpha_{2}\right), \sigma\left(\alpha_{2}+\right.\right.$ 2) $\}=\emptyset$.

Case 4-6: $m_{1}=m_{2}=4$.

Let $I\left(\boldsymbol{x}_{\pi(1)}\right)=\{\sigma(1)\}, I\left(\boldsymbol{x}_{\pi(2)}\right)=\{\sigma(2), \sigma(3)\}$, $I\left(\boldsymbol{x}_{\pi(3)}\right)=\{\sigma(4), \sigma(5)\}, \quad I\left(\boldsymbol{x}_{\pi(4)}\right)=\{\sigma(6), \sigma(7)\}$, $I\left(\boldsymbol{x}_{\pi(5)}\right)=\{\sigma(8), \sigma(10)\}, I\left(\boldsymbol{x}_{\pi(6)}\right)=\{\sigma(9), \sigma(11)\}$, $I\left(\boldsymbol{x}_{\pi(7)}\right)=\{\sigma(12), \sigma(14)\}$, and $I\left(\boldsymbol{x}_{\pi(8)}\right)=\{\sigma(13), \sigma(15)\}$.

Case 4-7: $m_{1}=5$ and $m_{2}=2$.

Case 4-7-1: $a_{1}=0$ and $a_{2}=1$.

Let $I\left(\boldsymbol{x}_{\pi(1)}\right)=\{\sigma(1)\}, I\left(\boldsymbol{x}_{\pi(2)}\right)=\{\sigma(4), \sigma(5)\}$, $I\left(\boldsymbol{x}_{\pi(3)}\right)=\{\sigma(6), \sigma(7)\}, I\left(\boldsymbol{x}_{\pi(4)}\right)=\{\sigma(\alpha), \sigma(\alpha+1)\}$, and $I\left(\boldsymbol{x}_{\pi(5)}\right)=\{\sigma(\alpha+2), \sigma(\alpha+3)\}$ such that $\alpha \in\{8,12\}$ and $A_{2} \cap\{\sigma(\alpha), \sigma(\alpha+1), \sigma(\alpha+2), \sigma(\alpha+3)\}=\emptyset$. Let $I\left(\boldsymbol{x}_{\pi(6)}\right)=\{\sigma(2)\}$ and $I\left(\boldsymbol{x}_{\pi(7)}\right)=\{\sigma(\beta), \sigma(\beta+2)\}$ such that $\beta \in\{8,9,12,13\}$ and $\left(A_{2} \cup\{\sigma(\alpha), \sigma(\alpha+1), \sigma(\alpha+\right.$ $2), \sigma(\alpha+3)\}) \cap\{\sigma(\beta), \sigma(\beta+2)\}=\emptyset$.
Case 4-7-2: $a_{1}=1$ and $a_{2}=0$.

Let $I\left(\boldsymbol{x}_{\pi(1)}\right)=\{\sigma(1)\}, I\left(\boldsymbol{x}_{\pi(2)}\right)=\{\sigma(8), \sigma(9)\}$, $I\left(\boldsymbol{x}_{\pi(3)}\right)=\{\sigma(10), \sigma(11)\}, I\left(\boldsymbol{x}_{\pi(4)}\right)=\{\sigma(12), \sigma(13)\}$, and $I\left(\boldsymbol{x}_{\pi(5)}\right)=\{\sigma(14), \sigma(15)\}$. Let $I\left(\boldsymbol{x}_{\pi(6)}\right)=\{\sigma(2)\}$ and $I\left(\boldsymbol{x}_{\pi(7)}\right)=\{\sigma(\alpha), \sigma(\alpha+2)\}$ such that $\alpha \in\{4,5\}$ and $A_{1} \cap\{\sigma(\alpha), \sigma(\alpha+2)\}=\emptyset$.

Case 4-8: $m_{1}=5$ and $m_{2}=3$.

Let $I\left(\boldsymbol{x}_{\pi(1)}\right)=\{\sigma(1)\}, I\left(\boldsymbol{x}_{\pi(2)}\right)=\{\sigma(4), \sigma(5)\}$, $I\left(\boldsymbol{x}_{\pi(3)}\right)=\{\sigma(6), \sigma(7)\}, I\left(\boldsymbol{x}_{\pi(4)}\right)=\{\sigma(8), \sigma(9)\}$, $I\left(\boldsymbol{x}_{\pi(5)}\right)=\{\sigma(10), \sigma(11)\}, I\left(\boldsymbol{x}_{\pi(6)}\right)=\{\sigma(2)\}, I\left(\boldsymbol{x}_{\pi(7)}\right)=$ $\{\sigma(12), \sigma(14)\}$, and $I\left(\boldsymbol{x}_{\pi(8)}\right)=\{\sigma(13), \sigma(15)\}$.

Case 4-9: $m_{1}=6$ and $m_{2}=2$.

Let $I\left(\boldsymbol{x}_{\pi(1)}\right)=\{\sigma(1)\}, I\left(\boldsymbol{x}_{\pi(2)}\right)=\{\sigma(2), \sigma(3)\}$, $I\left(\boldsymbol{x}_{\pi(3)}\right)=\{\sigma(4), \sigma(5)\}, I\left(\boldsymbol{x}_{\pi(4)}\right)=\{\sigma(6), \sigma(7)\}$, $I\left(\boldsymbol{x}_{\pi(5)}\right)=\{\sigma(8), \sigma(9)\}, I\left(\boldsymbol{x}_{\pi(6)}\right)=\{\sigma(10), \sigma(11)\}$, $I\left(\boldsymbol{x}_{\pi(7)}\right)=\{\sigma(12), \sigma(14)\}$, and $I\left(\boldsymbol{x}_{\pi(8)}\right)=\{\sigma(13), \sigma(15)\}$.

Case 5: There exist $2 \leq m_{1}, m_{2}, m_{3} \leq 4$, where $m_{1} \geq m_{2} \geq$ $m_{3}$, and the permutation $\pi$ of $\mathcal{I}_{8}$ such that $s_{\pi(1)}=\cdots=$ $\boldsymbol{s}_{\pi\left(m_{1}\right)}, \boldsymbol{s}_{\pi\left(m_{1}+1\right)}=\cdots=\boldsymbol{s}_{\pi\left(m_{1}+m_{2}\right)}, \boldsymbol{s}_{\pi\left(m_{1}+m_{2}+1\right)}=\cdots=$ $\boldsymbol{s}_{\pi\left(m_{1}+m_{2}+m_{3}\right)}$, and $\boldsymbol{s}_{\pi(i)} \neq \boldsymbol{s}_{\pi\left(i^{\prime}\right)}$ for any $i, i^{\prime} \in\left\{1, m_{1}+\right.$ $\left.1, m_{1}+m_{2}+1, m_{1}+m_{2}+m_{3}+1, \ldots, 8\right\}, i \neq i^{\prime}$.

Let $I\left(\boldsymbol{x}_{\pi\left(m_{1}+m_{2}+m_{3}+1\right)}\right)=\left\{j_{\pi\left(m_{1}+m_{2}+m_{3}+1\right)}\right\}, \ldots$, $I\left(\boldsymbol{x}_{\pi(8)}\right)=\left\{j_{\pi(8)}\right\}$.

Case 5-1: $\boldsymbol{s}_{\pi(1)}+\boldsymbol{s}_{\pi\left(m_{1}+1\right)} \neq \boldsymbol{s}_{\pi\left(m_{1}+m_{2}+1\right)}$.

From Theorem 5, we have the permutation $\sigma$ of $\mathcal{I}_{15}$ such that

$$
\begin{aligned}
& V\left(\boldsymbol{s}_{\pi(1)}\right) \\
= & \{\sigma(1)\},\{\sigma(2), \sigma(3)\},\{\sigma(4), \sigma(5)\}, \\
& \{\sigma(6), \sigma(7)\},\{\sigma(8), \sigma(9)\},\{\sigma(10), \sigma(11)\}, \\
& \{\sigma(12), \sigma(13)\},\{\sigma(14), \sigma(15)\}\}, \\
& V\left(\boldsymbol{s}_{\pi\left(m_{1}+1\right)}\right) \\
= & \{\{\sigma(2)\},\{\sigma(1), \sigma(3)\},\{\sigma(4), \sigma(6)\}, \\
& \{\sigma(5), \sigma(7)\},\{\sigma(8), \sigma(10)\},\{\sigma(9), \sigma(11)\}, \\
& \{\sigma(12), \sigma(14)\},\{\sigma(13), \sigma(15)\}\}, \\
& V\left(\boldsymbol{s}_{\pi\left(m_{1}+m_{2}+1\right)}\right) \\
= & \{\{\sigma(4)\},\{\sigma(1), \sigma(5)\},\{\sigma(2), \sigma(6)\}, \\
& \{\sigma(3), \sigma(7)\},\{\sigma(8), \sigma(12)\},\{\sigma(9), \sigma(13)\}, \\
& \{\sigma(10), \sigma(14)\},\{\sigma(11), \sigma(15)\}\} .
\end{aligned}
$$

Clearly, $\left\{j_{\pi\left(m_{1}+m_{2}+m_{3}+1\right)}, \ldots, j_{\pi(8)}\right\} \cap\{\sigma(1), \sigma(2), \sigma(4)\}=$ $\emptyset$. We define $A_{1}=\left\{j_{\pi(i)} \mid i \in\left\{m_{1}+m_{2}+m_{3}+\right.\right.$ $\left.1, \ldots, 8\}, j_{\pi(i)} \in\{\sigma(3), \sigma(5), \sigma(6), \sigma(7)\}\right\}, A_{2}=\left\{j_{\pi(i)}\right.$ $\left.i \in\left\{m_{1}+m_{2}+m_{3}+1, \ldots, 8\right\}, j_{\pi(i)} \in\{\sigma(8), \ldots, \sigma(15)\}\right\}$, $a_{1}=\left|A_{1}\right|, a_{2}=\left|A_{2}\right|$. Then, $a_{1}+a_{2}=8-m_{1}-m_{2}-m_{3}$.

Case 5-1-1: $m_{1}=m_{2}=m_{3}=2$.

Case 5-1-1-1: $a_{1}=0$ and $a_{2}=2$.

Let $I\left(\boldsymbol{x}_{\pi(1)}\right)=\{\sigma(1)\}, I\left(\boldsymbol{x}_{\pi(2)}\right)=\{\sigma(2), \sigma(3)\}$, $I\left(\boldsymbol{x}_{\pi(3)}\right)=\{\sigma(4), \sigma(6)\}$, and $I\left(\boldsymbol{x}_{\pi(4)}\right)=\{\sigma(5), \sigma(7)\}$. Let $I\left(\boldsymbol{x}_{\pi(5)}\right)=\left\{\sigma\left(\alpha_{1}\right), \sigma\left(\alpha_{1}+4\right)\right\}$ and $I\left(\boldsymbol{x}_{\pi(6)}\right)=$ $\left\{\sigma\left(\alpha_{2}\right), \sigma\left(\alpha_{2}+4\right)\right\}$ such that $\alpha_{1}, \alpha_{2} \in\{8,9,10,11\}, \alpha_{1} \neq \alpha_{2}$, and $A_{2} \cap\left\{\sigma\left(\alpha_{1}\right), \sigma\left(\alpha_{1}+4\right), \sigma\left(\alpha_{2}\right), \sigma\left(\alpha_{2}+4\right)\right\}=\emptyset$.

Case 5-1-1-2: $a_{1}=a_{2}=1$. 
Let $I\left(\boldsymbol{x}_{\pi(1)}\right)=\{\sigma(1)\}, I\left(\boldsymbol{x}_{\pi(3)}\right)=\{\sigma(2)\}$, and $I\left(\boldsymbol{x}_{\pi(5)}\right)=\{\sigma(4)\}$.

Case 5-1-1-2-1: $A_{2}=\{\sigma(8)\}$ or $A_{2}=\{\sigma(15)\}$.

Let $I\left(\boldsymbol{x}_{\pi(2)}\right)=\{\sigma(10), \sigma(11)\}, \quad I\left(\boldsymbol{x}_{\pi(4)}\right)=$ $\{\sigma(12), \sigma(14)\}$, and $I\left(\boldsymbol{x}_{\pi(6)}\right)=\{\sigma(9), \sigma(13)\}$.

Case 5-1-1-2-2: $A_{2}=\{\sigma(9)\}$ or $A_{2}=\{\sigma(14)\}$.

Let $I\left(\boldsymbol{x}_{\pi(2)}\right)=\{\sigma(12), \sigma(13)\}, I\left(\boldsymbol{x}_{\pi(4)}\right)=\{\sigma(8), \sigma(10)\}$, and $I\left(\boldsymbol{x}_{\pi(6)}\right)=\{\sigma(11), \sigma(15)\}$.

Case 5-1-1-2-3: $A_{2}=\{\sigma(10)\}$ or $A_{2}=\{\sigma(13)\}$.

Let $I\left(\boldsymbol{x}_{\pi(2)}\right)=\{\sigma(8), \sigma(9)\}, I\left(\boldsymbol{x}_{\pi(4)}\right)=\{\sigma(12), \sigma(14)\}$, and $I\left(\boldsymbol{x}_{\pi(6)}\right)=\{\sigma(11), \sigma(15)\}$.

Case 5-1-1-2-4: $A_{2}=\{\sigma(11)\}$ or $A_{2}=\{\sigma(12)\}$.

Let $I\left(\boldsymbol{x}_{\pi(2)}\right)=\{\sigma(14), \sigma(15)\}, I\left(\boldsymbol{x}_{\pi(4)}\right)=\{\sigma(8), \sigma(10)\}$, and $I\left(\boldsymbol{x}_{\pi(6)}\right)=\{\sigma(9), \sigma(13)\}$.

Case 5-1-1-3: $a_{1}=2$ and $a_{2}=0$.

Let $I\left(\boldsymbol{x}_{\pi(1)}\right)=\{\sigma(1)\}, I\left(\boldsymbol{x}_{\pi(2)}\right)=\{\sigma(8), \sigma(9)\}$, $I\left(\boldsymbol{x}_{\pi(3)}\right)=\{\sigma(2)\}, I\left(\boldsymbol{x}_{\pi(4)}\right)=\{\sigma(12), \sigma(14)\}, I\left(\boldsymbol{x}_{\pi(5)}\right)=$ $\{\sigma(4)\}$, and $I\left(\boldsymbol{x}_{\pi(6)}\right)=\{\sigma(11), \sigma(15)\}$.

Case 5-1-2: $m_{1}=3$ and $m_{2}=m_{3}=2$.

Case 5-1-2-1: $a_{1}=0$ and $a_{2}=1$.

Let $I\left(\boldsymbol{x}_{\pi(1)}\right)=\{\sigma(1)\}, I\left(\boldsymbol{x}_{\pi(2)}\right)=\{\sigma(4), \sigma(5)\}$, $I\left(\boldsymbol{x}_{\pi(3)}\right)=\{\sigma(6), \sigma(7)\}$, and $I\left(\boldsymbol{x}_{\pi(4)}\right)=\{\sigma(2)\}$.

Case 5-1-2-1-1: $A_{2}=\{\sigma(8)\}$ or $A_{2}=\{\sigma(10)\}$.

Let $I\left(\boldsymbol{x}_{\pi(5)}\right)=\{\sigma(12), \sigma(14)\}, I\left(\boldsymbol{x}_{\pi(6)}\right)=\{\sigma(9), \sigma(13)\}$, and $I\left(\boldsymbol{x}_{\pi(7)}\right)=\{\sigma(11), \sigma(15)\}$.

Case 5-1-2-1-2: $A_{2}=\{\sigma(9)\}$ or $A_{2}=\{\sigma(11)\}$.

Let $I\left(\boldsymbol{x}_{\pi(5)}\right)=\{\sigma(13), \sigma(15)\}, I\left(\boldsymbol{x}_{\pi(6)}\right)=\{\sigma(8), \sigma(12)\}$, and $I\left(\boldsymbol{x}_{\pi(7)}\right)=\{\sigma(10), \sigma(14)\}$.

Case 5-1-2-1-3: $A_{2}=\{\sigma(12)\}$ or $A_{2}=\{\sigma(14)\}$.

Let $I\left(\boldsymbol{x}_{\pi(5)}\right)=\{\sigma(8), \sigma(10)\}, I\left(\boldsymbol{x}_{\pi(6)}\right)=\{\sigma(9), \sigma(13)\}$, and $I\left(\boldsymbol{x}_{\pi(7)}\right)=\{\sigma(11), \sigma(15)\}$.

Case 5-1-2-1-4: $A_{2}=\{\sigma(13)\}$ or $A_{2}=\{\sigma(15)\}$.

Let $I\left(\boldsymbol{x}_{\pi(5)}\right)=\{\sigma(9), \sigma(11)\}, I\left(\boldsymbol{x}_{\pi(6)}\right)=\{\sigma(8), \sigma(12)\}$, and $I\left(\boldsymbol{x}_{\pi(7)}\right)=\{\sigma(10), \sigma(14)\}$.

Case 5-1-2-2: $a_{1}=1$ and $a_{2}=0$.

Let $I\left(\boldsymbol{x}_{\pi(1)}\right)=\{\sigma(1)\}, I\left(\boldsymbol{x}_{\pi(2)}\right)=\left\{\sigma\left(\alpha_{1}\right), \sigma\left(\alpha_{1}+1\right)\right\}$, and $I\left(\boldsymbol{x}_{\pi(3)}\right)=\left\{\sigma\left(\alpha_{2}\right), \sigma\left(\alpha_{2}+1\right)\right\}$ such that $\alpha_{1}, \alpha_{2} \in$ $\{2,4,6\}, \alpha_{1} \neq \alpha_{2}$, and $A_{1} \cap\left\{\sigma\left(\alpha_{1}\right), \sigma\left(\alpha_{1}+1\right), \sigma\left(\alpha_{2}\right), \sigma\left(\alpha_{2}+\right.\right.$ $1)\}=\emptyset$. Let $I\left(\boldsymbol{x}_{\pi(4)}\right)=\{\sigma(8), \sigma(10)\}, I\left(\boldsymbol{x}_{\pi(5)}\right)=$ $\{\sigma(12), \sigma(14)\}, I\left(\boldsymbol{x}_{\pi(6)}\right)=\{\sigma(9), \sigma(13)\}$, and $I\left(\boldsymbol{x}_{\pi(7)}\right)=$ $\{\sigma(11), \sigma(15)\}$.

Case 5-1-3: $m_{1}=m_{2}=3$ and $m_{3}=2$.

Let $I\left(\boldsymbol{x}_{\pi(1)}\right)=\{\sigma(1)\}, I\left(\boldsymbol{x}_{\pi(2)}\right)=\{\sigma(4), \sigma(5)\}$, $I\left(\boldsymbol{x}_{\pi(3)}\right)=\{\sigma(6), \sigma(7)\}, I\left(\boldsymbol{x}_{\pi(4)}\right)=\{\sigma(2)\}, I\left(\boldsymbol{x}_{\pi(5)}\right)=$ $\{\sigma(8), \sigma(10)\}, I\left(\boldsymbol{x}_{\pi(6)}\right)=\{\sigma(12), \sigma(14)\}, I\left(\boldsymbol{x}_{\pi(7)}\right)=$ $\{\sigma(9), \sigma(13)\}$, and $I\left(\boldsymbol{x}_{\pi(8)}\right)=\{\sigma(11), \sigma(15)\}$.

Case 5-1-4: $m_{1}=4$ and $m_{2}=m_{3}=2$.

Let $I\left(\boldsymbol{x}_{\pi(1)}\right)=\{\sigma(1)\}, I\left(\boldsymbol{x}_{\pi(2)}\right)=\{\sigma(2), \sigma(3)\}$, $I\left(\boldsymbol{x}_{\pi(3)}\right)=\{\sigma(4), \sigma(5)\}, I\left(\boldsymbol{x}_{\pi(4)}\right)=\{\sigma(6), \sigma(7)\}$, $I\left(\boldsymbol{x}_{\pi(5)}\right)=\{\sigma(8), \sigma(10)\}, I\left(\boldsymbol{x}_{\pi(6)}\right)=\{\sigma(12), \sigma(14)\}$, $I\left(\boldsymbol{x}_{\pi(7)}\right)=\{\sigma(9), \sigma(13)\}$, and $I\left(\boldsymbol{x}_{\pi(8)}\right)=\{\sigma(11), \sigma(15)\}$.

Case 5-2: $\boldsymbol{s}_{\pi(1)}+\boldsymbol{s}_{\pi\left(m_{1}+1\right)}=\boldsymbol{s}_{\pi\left(m_{1}+m_{2}+1\right)}$.
From Theorem 5, we have the permutation $\sigma$ of $\mathcal{I}_{15}$ such that

$$
\begin{aligned}
& V\left(\boldsymbol{s}_{\pi(1)}\right) \\
& =\{\{\sigma(1)\},\{\sigma(2), \sigma(3)\},\{\sigma(4), \sigma(5)\} \text {, } \\
& \{\sigma(6), \sigma(7)\},\{\sigma(8), \sigma(9)\},\{\sigma(10), \sigma(11)\} \text {, } \\
& \{\sigma(12), \sigma(13)\},\{\sigma(14), \sigma(15)\}\} \text {, } \\
& V\left(\boldsymbol{s}_{\pi\left(m_{1}+1\right)}\right) \\
& =\{\{\sigma(2)\},\{\sigma(1), \sigma(3)\},\{\sigma(4), \sigma(6)\} \text {, } \\
& \{\sigma(5), \sigma(7)\},\{\sigma(8), \sigma(10)\},\{\sigma(9), \sigma(11)\} \text {, } \\
& \{\sigma(12), \sigma(14)\},\{\sigma(13), \sigma(15)\}\} \text {, } \\
& V\left(\boldsymbol{s}_{\pi\left(m_{1}+m_{2}+1\right)}\right) \\
& =\{\{\sigma(3)\},\{\sigma(1), \sigma(2)\},\{\sigma(4), \sigma(7)\} \text {, } \\
& \{\sigma(5), \sigma(6)\},\{\sigma(8), \sigma(11)\},\{\sigma(9), \sigma(10)\}, \\
& \{\sigma(12), \sigma(15)\},\{\sigma(13), \sigma(14)\}\} \text {. }
\end{aligned}
$$

Clearly, $\left\{j_{\pi\left(m_{1}+m_{2}+m_{3}+1\right)}, \ldots, j_{\pi(8)}\right\} \cap\{\sigma(1), \sigma(2), \sigma(3)\}=$ $\emptyset$. We define $A_{1}=\left\{j_{\pi(i)} \mid i \in\left\{m_{1}+m_{2}+m_{3}+\right.\right.$ $\left.1, \ldots, 8\}, j_{\pi(i)} \in\{\sigma(4), \sigma(5), \sigma(6), \sigma(7)\}\right\}, A_{2}=\left\{j_{\pi(i)} \mid\right.$ $\left.i \in\left\{m_{1}+m_{2}+m_{3}+1, \ldots, 8\right\}, j_{\pi(i)} \in\{\sigma(8), \ldots, \sigma(15)\}\right\}$, $a_{1}=\left|A_{1}\right|, a_{2}=\left|A_{2}\right|$. Then, $a_{1}+a_{2}=8-m_{1}-m_{2}-m_{3}$.

Case 5-2-1: $m_{1}=m_{2}=m_{3}=2$.

Case 5-2-1-1: $a_{1}=0$ and $a_{2}=2$.

Let $I\left(\boldsymbol{x}_{\pi(1)}\right)=\{\sigma(1)\}, I\left(\boldsymbol{x}_{\pi(2)}\right)=\{\sigma(2), \sigma(3)\}$, $I\left(\boldsymbol{x}_{\pi(3)}\right)=\{\sigma(4), \sigma(6)\}$, and $I\left(\boldsymbol{x}_{\pi(4)}\right)=\{\sigma(5), \sigma(7)\}$. Let $I\left(\boldsymbol{x}_{\pi(5)}\right)=\left\{\sigma\left(\alpha_{1}\right), \sigma\left(\alpha_{1}^{\prime}\right)\right\}$ and $I\left(\boldsymbol{x}_{\pi(6)}\right)=$ $\left\{\sigma\left(\alpha_{2}\right), \sigma\left(\alpha_{2}^{\prime}\right)\right\}$ such that $\alpha_{1}, \alpha_{2} \in\{8,9,12,13\}, \alpha_{1}^{\prime}, \alpha_{2}^{\prime} \in$ $\{10,11,14,15\}, \alpha_{1} \neq \alpha_{2},\left\{\sigma\left(\alpha_{1}\right), \sigma\left(\alpha_{1}^{\prime}\right)\right\},\left\{\sigma\left(\alpha_{2}\right), \sigma\left(\alpha_{2}^{\prime}\right)\right\} \in$ $V\left(\boldsymbol{s}_{\pi(5)}\right)$, and $A_{2} \cap\left\{\sigma\left(\alpha_{1}\right), \sigma\left(\alpha_{1}^{\prime}\right), \sigma\left(\alpha_{2}\right), \sigma\left(\alpha_{2}^{\prime}\right)\right\}=\emptyset$.

Case 5-2-1-2: $a_{1}=a_{2}=1$.

Let $I\left(\boldsymbol{x}_{\pi(1)}\right)=\{\sigma(1)\}$ and $I\left(\boldsymbol{x}_{\pi(2)}\right)=\{\sigma(\alpha), \sigma(\alpha+1)\}$ such that $\alpha \in\{4,6\}$ and $A_{1} \cap\{\sigma(\alpha), \sigma(\alpha+1)\}=\emptyset$. Let $I\left(\boldsymbol{x}_{\pi(3)}\right)=\{\sigma(2)\}$ and $I\left(\boldsymbol{x}_{\pi(4)}\right)=\{\sigma(\beta), \sigma(\beta+2)\}$ such that $\beta \in\{8,9,12,13\}$ and $A_{2} \cap\{\sigma(\beta), \sigma(\beta+2)\}=\emptyset$. Let $I\left(\boldsymbol{x}_{\pi(5)}\right)=\{\sigma(3)\}$ and $I\left(\boldsymbol{x}_{\pi(6)}\right)=\left\{\sigma(\gamma), \sigma\left(\gamma^{\prime}\right)\right\}$ such that $\gamma \in\{8,9,12,13\}, \gamma^{\prime} \in\{10,11,14,15\},\left\{\sigma(\gamma), \sigma\left(\gamma^{\prime}\right)\right\} \in$ $V\left(s_{\pi(5)}\right)$, and $\left(A_{2} \cup\{\sigma(\beta), \sigma(\beta+2)\}\right) \cap\left\{\sigma(\gamma), \sigma\left(\gamma^{\prime}\right)\right\}=\emptyset$. Case 5-2-1-3: $a_{1}=2$ and $a_{2}=0$.

Let $I\left(\boldsymbol{x}_{\pi(1)}\right)=\{\sigma(1)\}, I\left(\boldsymbol{x}_{\pi(2)}\right)=\{\sigma(2), \sigma(3)\}$, $I\left(\boldsymbol{x}_{\pi(3)}\right)=\{\sigma(8), \sigma(10)\}, I\left(\boldsymbol{x}_{\pi(4)}\right)=\{\sigma(9), \sigma(11)\}$, $I\left(\boldsymbol{x}_{\pi(5)}\right)=\{\sigma(12), \sigma(15)\}$, and $I\left(\boldsymbol{x}_{\pi(6)}\right)=\{\sigma(13), \sigma(14)\}$.

Case 5-2-2: $m_{1}=3$ and $m_{2}=m_{3}=2$.

Case 5-2-2-1: $a_{1}=0$ and $a_{2}=1$.

Let $I\left(\boldsymbol{x}_{\pi(1)}\right)=\{\sigma(1)\}, I\left(\boldsymbol{x}_{\pi(2)}\right)=\{\sigma(4), \sigma(5)\}$, $I\left(\boldsymbol{x}_{\pi(3)}\right)=\{\sigma(6), \sigma(7)\}, I\left(\boldsymbol{x}_{\pi(4)}\right)=\{\sigma(2)\}$, and $I\left(\boldsymbol{x}_{\pi(5)}\right)=$ $\{\sigma(\alpha), \sigma(\alpha+2)\}$ such that $\alpha \in\{8,9\}$ and $A_{2} \cap\{\sigma(\alpha), \sigma(\alpha+$ $2)\}=\emptyset$. Let $I\left(\boldsymbol{x}_{\pi(6)}\right)=\{\sigma(3)\}$ and $I\left(\boldsymbol{x}_{\pi(7)}\right)=$ $\left\{\sigma(\beta), \sigma\left(\beta^{\prime}\right)\right\}$ such that $\beta \in\{12,13\}, \beta^{\prime} \in\{14,15\}$, $\left\{\sigma(\beta), \sigma\left(\beta^{\prime}\right)\right\} \in V\left(s_{\pi(6)}\right)$, and $A_{2} \cap\left\{\sigma(\beta), \sigma\left(\beta^{\prime}\right)\right\}=\emptyset$.

Case 5-2-2-2: $a_{1}=1$ and $a_{2}=0$.

Let $I\left(\boldsymbol{x}_{\pi(1)}\right)=\{\sigma(1)\}, I\left(\boldsymbol{x}_{\pi(2)}\right)=\{\sigma(2), \sigma(3)\}$, and $I\left(\boldsymbol{x}_{\pi(3)}\right)=\{\sigma(\alpha), \sigma(\alpha+1)\}$ such that $\alpha \in\{4,6\}$ and $A_{1} \cap\{\sigma(\alpha), \sigma(\alpha+1)\}=\emptyset$. Let $I\left(\boldsymbol{x}_{\pi(4)}\right)=\{\sigma(8), \sigma(10)\}$, 
$I\left(\boldsymbol{x}_{\pi(5)}\right)=\{\sigma(9), \sigma(11)\}, I\left(\boldsymbol{x}_{\pi(6)}\right)=\{\sigma(12), \sigma(15)\}$, and $I\left(\boldsymbol{x}_{\pi(7)}\right)=\{\sigma(13), \sigma(14)\}$.

Case 5-2-3: $m_{1}=m_{2}=3$ and $m_{3}=2$.

Let $I\left(\boldsymbol{x}_{\pi(1)}\right)=\{\sigma(1)\}, I\left(\boldsymbol{x}_{\pi(2)}\right)=\{\sigma(4), \sigma(5)\}$, $I\left(\boldsymbol{x}_{\pi(3)}\right)=\{\sigma(6), \sigma(7)\}, I\left(\boldsymbol{x}_{\pi(4)}\right)=\{\sigma(2)\}, I\left(\boldsymbol{x}_{\pi(5)}\right)=$ $\{\sigma(8), \sigma(10)\}, I\left(\boldsymbol{x}_{\pi(6)}\right)=\{\sigma(9), \sigma(11)\}, I\left(\boldsymbol{x}_{\pi(7)}\right)=$ $\{\sigma(3)\}$, and $I\left(\boldsymbol{x}_{\pi(8)}\right)=\{\sigma(12), \sigma(15)\}$.

Case 5-2-4: $m_{1}=4$ and $m_{2}=m_{3}=2$.

Let $I\left(\boldsymbol{x}_{\pi(1)}\right)=\{\sigma(1)\}, I\left(\boldsymbol{x}_{\pi(2)}\right)=\{\sigma(2), \sigma(3)\}$, $I\left(\boldsymbol{x}_{\pi(3)}\right)=\{\sigma(4), \sigma(5)\}, I\left(\boldsymbol{x}_{\pi(4)}\right)=\{\sigma(6), \sigma(7)\}$, $I\left(\boldsymbol{x}_{\pi(5)}\right)=\{\sigma(8), \sigma(10)\}, I\left(\boldsymbol{x}_{\pi(6)}\right)=\{\sigma(9), \sigma(11)\}$, $I\left(\boldsymbol{x}_{\pi(7)}\right)=\{\sigma(12), \sigma(15)\}$, and $I\left(\boldsymbol{x}_{\pi(8)}\right)=\{\sigma(13), \sigma(14)\}$.

Case 6: There exists the permutation $\pi$ of $\mathcal{I}_{8}$ such that $\boldsymbol{s}_{\pi(1)}=\boldsymbol{s}_{\pi(2)}, \boldsymbol{s}_{\pi(3)}=\boldsymbol{s}_{\pi(4)}, \boldsymbol{s}_{\pi(5)}=\boldsymbol{s}_{\pi(6)}, \boldsymbol{s}_{\pi(7)}=\boldsymbol{s}_{\pi(8)}$, and $\boldsymbol{s}_{\pi(i)} \neq \boldsymbol{s}_{\pi\left(i^{\prime}\right)}$ for any $i, i^{\prime} \in\{1,3,5,7\}, i \neq i^{\prime}$.

Case 6-1: $\boldsymbol{s}_{\pi(1)}, \boldsymbol{s}_{\pi(3)}, \boldsymbol{s}_{\pi(5)}$, and $\boldsymbol{s}_{\pi(7)}$ are linearly independent.

From Theorem 6, we have the permutation $\sigma$ of $\mathcal{I}_{15}$ such that

$$
\begin{aligned}
& V\left(s_{\pi(1)}\right) \\
= & \{\sigma(1)\},\{\sigma(2), \sigma(3)\},\{\sigma(4), \sigma(5)\}, \\
& \{\sigma(6), \sigma(7)\},\{\sigma(8), \sigma(9)\},\{\sigma(10), \sigma(11)\}, \\
& \{\sigma(12), \sigma(13)\},\{\sigma(14), \sigma(15)\}\}, \\
& V\left(s_{\pi(3)}\right) \\
= & \{\{\sigma(2)\},\{\sigma(1), \sigma(3)\},\{\sigma(4), \sigma(6)\}, \\
& \{\sigma(5), \sigma(7)\},\{\sigma(8), \sigma(10)\},\{\sigma(9), \sigma(11)\}, \\
& \{\sigma(12), \sigma(14)\},\{\sigma(13), \sigma(15)\}\}, \\
& V\left(s_{\pi(5)}\right) \\
= & \{\sigma(4)\},\{\sigma(1), \sigma(5)\},\{\sigma(2), \sigma(6)\}, \\
& \{\sigma(3), \sigma(7)\},\{\sigma(8), \sigma(12)\},\{\sigma(9), \sigma(13)\}, \\
& \{\sigma(10), \sigma(14)\},\{\sigma(11), \sigma(15)\}\}, \\
& V\left(s_{\pi(7)}\right) \\
= & \{\sigma(8)\},\{\sigma(1), \sigma(9)\},\{\sigma(2), \sigma(10)\}, \\
& \{\sigma(3), \sigma(11)\},\{\sigma(4), \sigma(12)\},\{\sigma(5), \sigma(13)\}, \\
& \{\sigma(6), \sigma(14)\},\{\sigma(7), \sigma(15)\}\} .
\end{aligned}
$$

Let $I\left(\boldsymbol{x}_{\pi(1)}\right)=\{\sigma(1)\}, I\left(\boldsymbol{x}_{\pi(2)}\right)=\{\sigma(2), \sigma(3)\}, I\left(\boldsymbol{x}_{\pi(3)}\right)=$ $\{\sigma(4), \sigma(6)\}, I\left(\boldsymbol{x}_{\pi(4)}\right)=\{\sigma(9), \sigma(11)\}, I\left(\boldsymbol{x}_{\pi(5)}\right)=$ $\{\sigma(8), \sigma(12)\}, I\left(\boldsymbol{x}_{\pi(6)}\right)=\{\sigma(10), \sigma(14)\}, I\left(\boldsymbol{x}_{\pi(7)}\right)=$ $\{\sigma(5), \sigma(13)\}$, and $I\left(\boldsymbol{x}_{\pi(8)}\right)=\{\sigma(7), \sigma(15)\}$.

Case 6-2: There exists the permutation $\tau$ of $\{\pi(1), \pi(3), \pi(5), \pi(7)\}$ such that $\boldsymbol{s}_{\tau(\pi(1))}+\boldsymbol{s}_{\tau(\pi(3))}=$ $\boldsymbol{s}_{\tau(\pi(5))}$.

For simplicity, suppose $\boldsymbol{s}_{\pi(1)}+\boldsymbol{s}_{\pi(3)}=\boldsymbol{s}_{\pi(5)}$. From Theorem 6 , we have the permutation $\sigma$ of $\mathcal{I}_{15}$ such that

$$
\begin{aligned}
& V\left(\boldsymbol{s}_{\pi(1)}\right) \\
= & \{\{\sigma(1)\},\{\sigma(2), \sigma(3)\},\{\sigma(4), \sigma(5)\}, \\
& \{\sigma(6), \sigma(7)\},\{\sigma(8), \sigma(9)\},\{\sigma(10), \sigma(11)\}, \\
& \{\sigma(12), \sigma(13)\},\{\sigma(14), \sigma(15)\}\}, \\
& V\left(s_{\pi(3)}\right)
\end{aligned}
$$

$$
\begin{aligned}
= & \{\{\sigma(2)\},\{\sigma(1), \sigma(3)\},\{\sigma(4), \sigma(6)\}, \\
& \{\sigma(5), \sigma(7)\},\{\sigma(8), \sigma(10)\},\{\sigma(9), \sigma(11)\}, \\
& \{\sigma(12), \sigma(14)\},\{\sigma(13), \sigma(15)\}\}, \\
& V\left(s_{\pi(5)}\right) \\
= & \{\{\sigma(3)\},\{\sigma(1), \sigma(2)\},\{\sigma(4), \sigma(7)\}, \\
& \{\sigma(5), \sigma(6)\},\{\sigma(8), \sigma(11)\},\{\sigma(9), \sigma(10)\}, \\
& \{\sigma(12), \sigma(15)\},\{\sigma(13), \sigma(14)\}\}, \\
& V\left(s_{\pi(7)}\right) \\
= & \{\{\sigma(4)\},\{\sigma(1), \sigma(5)\},\{\sigma(2), \sigma(6)\}, \\
& \{\sigma(3), \sigma(7)\},\{\sigma(8), \sigma(12)\},\{\sigma(9), \sigma(13)\}, \\
& \{\sigma(10), \sigma(14)\},\{\sigma(11), \sigma(15)\}\} .
\end{aligned}
$$

Let $I\left(\boldsymbol{x}_{\pi(1)}\right)=\{\sigma(1)\}, I\left(\boldsymbol{x}_{\pi(2)}\right)=\{\sigma(2), \sigma(3)\}, I\left(\boldsymbol{x}_{\pi(3)}\right)=$ $\{\sigma(4), \sigma(6)\}, I\left(\boldsymbol{x}_{\pi(4)}\right)=\{\sigma(5), \sigma(7)\}, I\left(\boldsymbol{x}_{\pi(5)}\right)=$ $\{\sigma(8), \sigma(11)\}, I\left(\boldsymbol{x}_{\pi(6)}\right)=\{\sigma(12), \sigma(15)\}, I\left(\boldsymbol{x}_{\pi(7)}\right)=$ $\{\sigma(9), \sigma(13)\}$, and $I\left(\boldsymbol{x}_{\pi(8)}\right)=\{\sigma(10), \sigma(14)\}$.

Case 6-3: $\boldsymbol{s}_{\pi(1)}+\boldsymbol{s}_{\pi(3)}=\boldsymbol{s}_{\pi(5)}+\boldsymbol{s}_{\pi(7)}$.

From Theorem 6, we have the permutation $\sigma$ of $\mathcal{I}_{15}$ such that

$$
\begin{aligned}
& V\left(s_{\pi(1)}\right) \\
= & \{\{\sigma(1)\},\{\sigma(2), \sigma(3)\},\{\sigma(4), \sigma(5)\}, \\
& \{\sigma(6), \sigma(7)\},\{\sigma(8), \sigma(9)\},\{\sigma(10), \sigma(11)\}, \\
& \{\sigma(12), \sigma(13)\},\{\sigma(14), \sigma(15)\}\}, \\
& V\left(s_{\pi(3)}\right) \\
= & \{\{\sigma(2)\},\{\sigma(1), \sigma(3)\},\{\sigma(4), \sigma(6)\}, \\
& \{\sigma(5), \sigma(7)\},\{\sigma(8), \sigma(10)\},\{\sigma(9), \sigma(11)\}, \\
& \{\sigma(12), \sigma(14)\},\{\sigma(13), \sigma(15)\}\}, \\
& V\left(s_{\pi(5)}\right) \\
= & \{\sigma(4)\},\{\sigma(1), \sigma(5)\},\{\sigma(2), \sigma(6)\}, \\
& \{\sigma(3), \sigma(7)\},\{\sigma(8), \sigma(12)\},\{\sigma(9), \sigma(13)\}, \\
& \{\sigma(10), \sigma(14)\},\{\sigma(11), \sigma(15)\}\}, \\
& V\left(s_{\pi(7)}\right) \\
= & \{\{\sigma(7)\},\{\sigma(1), \sigma(6)\},\{\sigma(2), \sigma(5)\}, \\
& \{\sigma(3), \sigma(4)\},\{\sigma(8), \sigma(15)\},\{\sigma(9), \sigma(14)\}, \\
& \{\sigma(10), \sigma(13)\},\{\sigma(11), \sigma(12)\}\} .
\end{aligned}
$$

Let $I\left(\boldsymbol{x}_{\pi(1)}\right)=\{\sigma(1)\}, I\left(\boldsymbol{x}_{\pi(2)}\right)=\{\sigma(2), \sigma(3)\}, I\left(\boldsymbol{x}_{\pi(3)}\right)=$ $\{\sigma(4), \sigma(6)\}, \quad I\left(\boldsymbol{x}_{\pi(4)}\right)=\{\sigma(5), \sigma(7)\}, I\left(\boldsymbol{x}_{\pi(5)}\right)=$ $\{\sigma(8), \sigma(12)\}, I\left(\boldsymbol{x}_{\pi(6)}\right)=\{\sigma(11), \sigma(15)\}, I\left(\boldsymbol{x}_{\pi(7)}\right)=$ $\{\sigma(9), \sigma(14)\}$, and $I\left(\boldsymbol{x}_{\pi(8)}\right)=\{\sigma(10), \sigma(13)\}$.

Therefore, from Theorem 2] the $[15,4,8] \mathrm{P}-\mathrm{RIO}$ code can be constructed using the coset coding. The number of pages stored by this code is greater than that of the $[15,4,6]$ RIO code constructed using the same $(15,11)$ Hamming code.

An upper bound on the sum-rate of RIO codes and P-RIO codes that store $t$ pages is $\log (t+1)$ [3]. Table IV] shows the sum-rates and the upper bounds on that of the RIO codes and the P-RIO codes constructed via coset coding with Hamming codes. 
TABLE IV

COMPARISON WITH CAPACITY

\begin{tabular}{|c|c|c|c|c|}
\hline \multirow{2}{*}{ Code length } & \multicolumn{2}{|c|}{ RIO code } & \multicolumn{2}{c|}{ P-RIO code } \\
\cline { 2 - 5 } & Sum-rate & Upper bound & Sum-rate & Upper bound \\
\hline 7 & 1.2857 & 2 & 1.7142 & 2.3219 \\
\hline 15 & 1.6 & 2.8073 & 2.1333 & 3.1699 \\
\hline
\end{tabular}

\section{CONCLUSION}

In this paper, we have constructed P-RIO codes using coset coding with Hamming codes. When each page is encoded, the information on the data of the other pages is leveraged to increase the number of stored pages compared to that for RIO codes. Our P-RIO codes are constructive, whereas the approach in [3] is based on exhaustive search. However, in our approach, the number of cases required for the encoding increases with the number of pages. Therefore, we should consider another approach to deriving the number of pages of P-RIO codes that can be constructed using Hamming codes of length $\left(2^{r}-1\right)$ for $r \geq 5$.

\section{ACKNOWLEDGMENT}

This work was partially supported by the Advanced Storage Research Consortium and JSPS KAKENHI Grant Number $15 \mathrm{~K} 00010$.

\section{REFERENCES}

[1] E. Sharon and I. Alrod, "Coding scheme for optimizing random I/O performance," in Proc. Non-Volatile Memories Workshop, San Diego, CA, USA, Apr. 2013.

[2] R. L. Rivest and A. Shamir, "How to reuse a write-once memory," Inf. Control, vol. 55, no. 1-3, pp. 1-19, Dec. 1982.

[3] E. Yaakobi and R. Motwani, "Construction of random input-output codes with moderate block lengths," IEEE Trans. Commun., vol. 64, no. 5, May 2016.

[4] G. D. Cohen, P. Godlewski, and F. Merkx, "Linear binary code for write-once memories," IEEE Trans. Inf. Theory, vol. IT-32, no. 5, pp. 697-700, Oct. 1986.

[5] N. Okamae, T. Fujiwara, and Y. Kaji "A floating code construction using coset coding with Hamming code" (in Japanese), Proc. 35th Symposium on Information Theory and its Applications, pp. 578-583, Oita, Japan, December 2012. 